\title{
Macroscopic constitutive relations for elastomers reinforced with short aligned fibers: instabilities and post-bifurcation response
}

\author{
Reza Avazmohammadi ${ }^{\mathrm{a}}$, Pedro Ponte Castañeda ${ }^{\mathrm{a}, *}$ \\ ${ }^{a}$ Department of Mechanical Engineering and Applied Mechanics, University of Pennsylvania, \\ Philadelphia, PA 19104-6315, U.S.A.
}

\begin{abstract}
This paper is concerned with the characterization of the macroscopic response and possible development of instabilities in a certain class of anisotropic composite materials consisting of random distributions of aligned rigid fibers of elliptical cross section in a soft elastomeric matrix, which are subjected to general plane strain loading conditions. For this purpose, use is made of an estimate for the stored-energy function that was derived by Lopez-Pamies and Ponte Castañeda (2006b) for this class of reinforced elastomers by means of the second-order linear comparison homogenization method. This homogenization estimate has been shown to lose strong ellipticity by the development of shear localization bands, when the composite is loaded in compression along the (in-plane) long axes of the fibers. The instability is produced by the sudden, collective rotation of a band of fibers to partially release the high stresses that develop in the elastomer matrix when the composite is compressed along the stiff, long-fiber direction. Consistent with the mode of the impending instability, a lower-energy, post-bifurcation solution is constructed where "striped domain" microstructures consisting of layers with alternating fiber orientations develop in the composite. The volume fractions of the layers and the fiber orientations within the layers adjust themselves to satisfy equilibrium and compatibility across the layers, while remaining compatible with the imposed overall deformation. Mathematically, this construction is shown to correspond to the rank-one convex envelope of the original estimate
\end{abstract}

\footnotetext{
${ }^{*}$ Corresponding author

Email addresses: rezaavaz@seas . upenn. edu (Reza Avazmohammadi), ponte@seas . upenn . edu (Pedro Ponte Castañeda)
} 
for the energy, and is further shown to be polyconvex and therefore quasiconvex. Thus, it corresponds to the "relaxation" of the stored-energy function of the composite, and can in turn be viewed as a stress-driven "phase transition," where the symmetry of the fiber microstructures changes from nematic to smectic.

Keywords: Hyperelastic composites, Finite deformations, Mechanical instabilities

\section{Introduction}

Fiber-reinforced elastomers are a special class of hyperelastic composite materials consisting of stiff fibers that are distributed randomly or periodically in an soft elastomeric matrix material that can undergo finite deformations. The characterization of the macroscopic behavior of these composites is a central problem in mechanics of materials, in particular, because they are ubiquitous both in manmade and naturally appearing material systems. Examples of man-made systems include reinforced rubbers for car tire and deployable structure applications, as well as thermoplastic elastomers for numerous other applications, while arterial walls and other connective fibrous tissues provide examples of natural systems. Depending on the length of the fibers, reinforced elastomers can be classified as short-fiber-reinforced elastomers (O'Connor, 1977), and long- or continuous-fiber elastomers (Spencer, 1984; Honeker and Thomas, 1996).

The basic homogenization framework for the theoretical characterization of the macroscopic response of hyperelastic composites undergoing finite deformations dates back to the work of Hill (1972), generalizing earlier work for linearelastic composites (see, for example, Willis, 1981). However, due to the difficulties associated with the significant constitutive and geometric nonlinearities involved, progress in this field has been slow, at least compared to corresponding progress for other nonlinear composite materials, such as viscoplastic composites (Ponte Castañeda and Suquet, 1998). Microstructure-independent, Voigt-type bounds on the homogenized stored-energy function of hyperelastic composites were proposed by Ogden (1978). Exact results for layered composites under special plane boundary conditions were given by Triantafyllidis and Maker (1985). On the other hand, exact results for porous elastomers with special compositesphere microstructures and hydrostatic loading conditions were derived by Hashin (1985), while incremental numerical homogenization calculations for two-dimensional porous elastomers were developed by Abeyaratne and Triantafyllidis (1984). Lower bounds on the homogenized stored-energy function were proposed by Ponte Castañeda 
(1989) exploiting the polyconvexity hypothesis (Ball, 1977).

General techniques for homogenizing hyperelastic composites in the finite strain regime did not become available until the turn of the century. Building on earlier work for viscoplastic composites (Ponte Castañeda, 1991, 1996, 2002), variational techniques making use of suitably chosen linear comparison composites were proposed by Ponte Castañeda and Tiberio (2000) and Lopez-Pamies and Ponte Castañeda (2004). These "second-order" methods have the property that they yield estimates that are exact to second-order in the heterogeneity contrast (Suquet and Ponte Castañeda, 1993). They have been applied to estimate the transverse shear response of fiber-reinforced elastomers with random and periodic microstructures by Ponte Castañeda and Tiberio (2000) and Lahellec et al. (2004), respectively. More general estimates for the transverse response of elastomers reinforced by fibers with elliptical cross-section have been given by Lopez-Pamies and Ponte Castañeda (2006a,b) and Chen et al. (2011). In the earlier work, it was observed that the in-plane rotation of the fibers is an important variable that can lead to significant geometric softening under certain loading conditions. Consideration of the full three-dimensional response of elastomers reinforced with periodic and random distributions of (aligned) cylindrical fibers was given by Brun et al. (2007) and Agoras et al. (2009a), respectively. These results where found to be in reasonably good agreement with numerical simulations (Moraleda et al., 2009; Bouchart et al., 2010), as well as with estimates derived by an alternative homogenization approach (deBotton, 2005; deBotton et al., 2006). Elastomers reinforced with spherical and aligned spheroidal fibers have been recently considered by Avazmohammadi and Ponte Castañeda (2013, 2014a,b). In addition, building on exact results for layered composites by deBotton (2005), Triantafyllidis et al. (2006) and Lopez-Pamies and Ponte Castañeda (2009), applications to thermoplastic elastomers with oriented and unoriented polydomain layered morphologies have been considered by Lopez-Pamies et al. (2008) and Racherla et al. (2010). Finally, it should also be mentioned that numerical homogenization schemes have been developed by Miehe et al. (2002) for hyperelastic composites. Additional numerical homogenization results for fiber-reinforced elastomers have been provided by Guo et al. (2007) and Jimenez and Pellegrino (2012).

One distinguishing feature of hyperelastic composites arising from the intrinsic non-convexity of their stored-energy functions is that they may undergo instabilities where the unique "principal" solution that is observed at low deformations bifurcates into other, lower energy solutions at sufficiently large deformations. Thus, in the above-mentioned work of Triantafyllidis and Maker (1985) for layered hyperelastic composites with periodic microstructures, it was shown that mi- 
crobuckling instabilities are possible in these materials when they are compressed along the layer direction, where solutions with uniform fields in the layers give way to lower-energy solutions with non-uniform deformations in the layers. In addition, it was found in that work that it is often the case that the first instability corresponds to a long (infinite) wavelength mode. Moreover, the critical stretch at these long wavelength instabilities can be captured by the loss of strong ellipticity of the homogenized response of the composite. Remarkably, it was further observed that loss of strong ellipticity of the macroscopic response of the composite could still take place when the properties of the constituent phases are themselves strongly elliptic.

For hyperelastic composites with more general periodic microstructures, mathematically rigorous definitions of the effective energy $\widetilde{W}$ have been given by Braides (1985) and Müller (1987). Such definitions generalize the classical definition of the effective energy for periodic media with convex energies (Marcellini, 1978), by accounting for the fact that, in the non-convex case, it is not sufficient to consider one-cell periodic solutions, as solutions involving interactions between several unit cells may lead to lower macroscopic energies. This corresponds to the possible development of "microscopic" instabilities in the composite at sufficiently high deformation, consisting of solutions that are periodic on $N \times M \times L$ super-cells. However, before the onset of such instabilities, $\widetilde{W}$ is given by the one-cell periodic solution, which may be computed from the effective energy associated with one unit cell (with periodic boundary conditions), just as in the case when the local energy function is convex. In addition, it is important to remark that Geymonat et al. (1993), building on earlier work (Abeyaratne and Triantafyllidis, 1984; Triantafyllidis and Maker, 1985), have shown rigorously that loss of strong ellipticity in the homogenized behavior of the composite corresponds to the development of long-wavelength, or "macroscopic" instabilities in the form of localized shear bands. Furthermore, it is often the case (Geymonat et al., 1993; Triantafyllidis et al., 2006) that the first instability is indeed a long-wavelength instability, in which case $\widetilde{W}$ may be easily computed from one unit-cell periodic solutions all the way up to the development of a macroscopic instability. Thus, the macroscopic instabilities may be obtained from the one-cell periodic solutions, and are therefore much easier to compute than the microscopic instabilities. More generally, however, the first instability is of finite wavelength (i.e., small compared to the size of the specimen), but even in this case, the loss of strong ellipticity of the homogenized energy provides an upper bound for the development of microscopic instabilities. In other words, the composite material will become unstable before reaching the "failure surface" defined by the macroscopic instabil- 
ities (Triantafyllidis et al., 2006). The possible development of "microscopic" and "macroscopic" instabilities in two-dimensional porous elastomers and reinforced elastomers with periodic microstructures has been investigated recently by Triantafyllidis et al. (2006), Michel et al. (2007) and Michel et al. (2010). In fact, one of the instabilities in the porous elastomers, where the one-cell periodic solution with initially circular holes bifurcates into a lower-energy solution with elliptical holes in a $2 \times 2$ pattern with alternating orientations for the ellipses-which was first reported in Triantafyllidis et al. (2006), and developed fully in Michel et al. (2007) — has been observed experimentally (Mullin et al., 2007) and in numerical simulations (Bertoldi et al., 2007). For layered microstructures, Miehe et al. (2002) have also generated numerical estimates for the post-buckling response.

For hyperelastic composites with random microstructures, the theory is less developed. However, it has been argued by Lopez-Pamies and Ponte Castañeda (2004) and Michel et al. (2007) in the context of porous elastomers that while "microscopic" instabilities are not expected to survive in the random case, the long-wavelength "macroscopic" instabilities should still be relevant for random microstructures, and can still be computed from the homogenized response of the composite. For elastomers reinforced with aligned elliptical fibers subjected to plane deformations, it has been shown by Lopez-Pamies and Ponte Castañeda $(2006 a, b)$ that the homogenized response of the composite can lose strong ellipticity, due to the softening associated with the fiber rotations. When the composite is loaded in such a way that the long axes of the elliptical fibers experience compression, the high stress that develop as a consequence can be relieved by the sudden collective rotation of the fibers, leading to a so-called "flopping" instability. Loss of strong ellipticity of the homogenized response was also found by Brun et al. (2007) and Agoras et al. (2009b) for elastomers reinforced by periodic and random distributions of aligned cylindrical fibers under general three-dimensional loading conditions. Very recently, Avazmohammadi and Ponte Castañeda (2014a,b) have similarly found loss of strong ellipticity for elastomers reinforced by randomly positioned, but aligned spheroidal fibers under various loading conditions involving compression along the long axes of the fibers. In addition, loss of strong ellipticity has been found by Lopez-Pamies and Ponte Castañeda (2007a,b) for porous elastomers with initially isotropic distributions of spherical voids.

As we have seen, homogenization techniques are available which allow the estimation of the macroscopic response of hyperelastic composites with random microstructures. In addition, the possible development of macroscopic instabilities in such composites can be captured consistently from the loss of strong ellipticity of the homogenized response. However, it is clear that such estimates for 
the homogenized response cannot continue to be used beyond the onset of such macroscopic instabilities. It is the objective of this work to make a first attempt to go beyond the macroscopic instabilities into the post-bifurcation regime. For this purpose, we will work in the context of the two-dimensional problem defined by Lopez-Pamies and Ponte Castañeda (2006b) involving elastomers that are reinforced by aligned fibers with elliptical cross section and are subjected to general plane strain loading conditions. Guided by the mathematical theory developed by Müller (1987) and Geymonat et al. (1993), and building on successful applications in the context of other material systems, such as shape-memory alloys (Ball and James, 1986), ferromagnetic crystals (DeSimone, 1993) and nematic liquid crystal elastomers (DeSimone and Dolzmann, 2002), we will undertake here the computation of the quasiconvexification of the estimate developed by Lopez-Pamies and Ponte Castañeda (2006b) for the effective stored-energy function. Thus, we will show that such a relaxation of the energy actually corresponds to the rank-one convex envelope of the energy, and is consistent with the shear localization instabilities associated with loss of strong ellipticity of the estimates of Lopez-Pamies and Ponte Castañeda (2006b) for the effective energy.

The rest of the paper is organized as follows. In Section 2, we provide some general preliminaries on homogenization, including some basic constitutive hypotheses on the local energy functions of the composites, as well as the definitions of the "principal" and "post-bifurcation" solutions for the macroscopic storedenergy functions. We also argue that the relaxed homogenized energy corresponds to the quasiconvexification of the principal solution of the energy. In Section 3, the fiber-reinforced elastomers and loading conditions of interest are defined, and the corresponding estimate of Lopez-Pamies and Ponte Castañeda (2006b) for the stored-energy function is recalled and re-expressed in various forms. The basic features of the principal solution are identified, including the evolution of the fiber orientation with the deformation, and its implications for loss of strong ellipticity. In Section 4, we compute the relaxation of the energy from the principal energy in two steps. We first compute the rank-one convexification of this energy and we then show (in an appendix) that it is polyconvex, and therefore quasi-convex. The main features of the relaxed solution are identified, including the characterization of the microstructures that arise beyond the loss of ellipticity of the principal solution, as well as the soft modes of deformation resulting as a consequence of these instabilities. In addition, the dependence of these microscopic and macroscopic features on the microstructural parameters, such as the fiber volume fraction and shape, as well as the elastomer constitutive properties are investigated. Finally, some general conclusions will be drawn. 


\section{Hyperelastic composite elastomers at finite deformation: Preliminaries}

For simplicity, we consider here composites made up of only two different (homogeneous) phases, which are distributed randomly in a specimen occupying a volume $\Omega_{0}$ in the reference configuration, where the characteristic length of the inhomogeneities (e.g., particles, fibers, etc.) is assumed to be much smaller than the size of the specimen and the scale of variation of the applied loading. Thus, in the homogenization limit (i.e., in the limit as the size of the inhomogeneities tends to zero), $\Omega_{0}$ can be identified with a representative volume element (RVE) of the composite elastomer. The position vector of a material point in the undeformed configuration $\Omega_{0}$ is denoted by $\mathbf{X}$, with Cartesian components $X_{i}(i=1,2,3)$ and the corresponding position vector in the deformed configuration $\Omega$ is denoted by $\mathbf{x}$, with components $x_{i}(i=1,2,3)$. The deformation gradient tensor $\mathbf{F}$ has components $F_{i j}=\partial x_{i} / \partial X_{j}$ and is required to satisfy the material impenetrability condition: $J=\operatorname{det}(\mathbf{F})>0$ for all $\mathbf{X} \in \Omega_{0}$.

\subsection{Standard homogenization framework}

The constitutive behavior of the phases is characterized by stored-energy functions $W^{(r)}(r=1,2)$, which are taken to be non-convex functions of the deformation gradient tensor $\mathbf{F}$. These stored-energy functions are, of course, assumed to be objective, so that $W^{(r)}(\mathbf{Q F})=W^{(r)}(\mathbf{F})$ for all proper orthogonal $\mathbf{Q}$ and arbitrary deformation gradients $\mathbf{F}$. In particular, by making use of the polar decomposition $\mathbf{F}=\mathbf{R} \mathbf{U}$, where $\mathbf{U}$ is the right-stretch tensor and $\mathbf{R}$ is the rotation tensor, it follows that $W^{(r)}(\mathbf{F})=W^{(r)}(\mathbf{U})$.

The local stored-energy function of the hyperelastic composite can then be expressed as

$$
W(\mathbf{X}, \mathbf{F})=\sum_{r=1}^{2} \chi_{0}^{(r)}(\mathbf{X}) W^{(r)}(\mathbf{F}),
$$

where the characteristic functions $\chi_{0}^{(r)}$ are equal to 1 , if the position vector $\mathbf{X}$ is inside phase $r$ (i.e., $\mathbf{X} \in \Omega_{0}^{(r)}$ ), and zero otherwise. Note that since $\chi_{0}^{(1)}+\chi_{0}^{(2)}=1$ we can let $\chi_{0}^{(2)}=\chi_{0}$, so that $\chi_{0}^{(1)}=1-\chi_{0}$. The characteristic function $\chi_{0}$ may be periodic or random. In the first case, the dependence of $\chi_{0}$ on the position vector $\mathbf{X}$ is completely determined once a unit cell $D$ has been specified. In the second case, the dependence of $\chi_{0}$ on $\mathbf{X}$ is not known precisely, but under the assumption of statistical uniformity, the microstructure can be partially characterized in terms of the $n$-point correlation functions. Here, we will focus on random microstructures and use will be made of information only up to two-point statistics in order to be 
able to take advantage of linear homogenization estimates that are available from the literature (Ponte Castañeda and Willis, 1995). It is noted that the initial volume fraction of phase 2 is given by $c_{0}^{(2)}=c_{0}=\left\langle\chi_{0}\right\rangle$, where the triangular brackets denote volume averages (in the undeformed configuration) over the volume $\Omega_{0}$, that is

$$
\left\langle\chi_{0}\right\rangle \equiv \frac{1}{\Omega_{0}} \int_{\Omega_{0}} \chi_{0}(\mathbf{X}) d V_{\mathbf{X}} .
$$

Of course, the initial volume fraction of phase 1 is given by $1-c_{0}$.

The local or microscopic constitutive relation for the composite is then given by

$$
\mathbf{S}=\frac{\partial W}{\partial \mathbf{F}}(\mathbf{X}, \mathbf{F})
$$

where $\mathbf{S}$ denotes the first Piola-Kirchhoff stress tensor. Assuming sufficient smoothness for $W$ in $\mathbf{F}$, it is also useful to define the local elasticity, or tangent modulus tensor of the composite material via

$$
\mathbf{L}(\mathbf{X}, \mathbf{F}) \equiv \frac{\partial^{2} W}{\partial \mathbf{F} \partial \mathbf{F}}(\mathbf{X}, \mathbf{F})
$$

Now, following Hill (1972), the effective stored-energy function $\widetilde{W}$ of the composite elastomer is defined by

$$
\widetilde{W}(\overline{\mathbf{F}})=\min _{\mathbf{F} \in \mathscr{K}(\overline{\mathbf{F}})}\langle W(\mathbf{X}, \mathbf{F})\rangle
$$

where $\mathscr{K}(\overline{\mathbf{F}})$ denotes the set of kinematically admissible deformation gradients:

$$
\mathscr{K}(\overline{\mathbf{F}})=\left\{\mathbf{F} \mid \exists \mathbf{x}=\mathbf{x}(\mathbf{X}), \text { with } \mathbf{F}=\operatorname{Grad} \mathbf{x} \text { in } \Omega_{0}, \mathbf{x}=\overline{\mathbf{F}} \mathbf{X} \text { on } \partial \Omega_{0}\right\} .
$$

(The constraint $J=\operatorname{det}(\mathbf{F})>0$ will be enforced by the choice of appropriate forms for the phase energy functions $W^{(r)}$.) It is noted that $\widetilde{W}$ physically represents the average elastic energy that is stored in the composite when it is subjected to an affine displacement on its boundary with prescribed deformation gradient $\overline{\mathbf{F}}$. Moreover, from the definition (5) and the objectivity of $W^{(r)}$, it can be shown that $\widetilde{W}$ is objective, namely, $\widetilde{W}(\overline{\mathbf{F}})=\widetilde{W}(\overline{\mathbf{U}})$. Here, $\overline{\mathbf{U}}$ represents the macroscopic right-stretch tensor associated with the macroscopic polar decomposition $\overline{\mathbf{F}}=\overline{\mathbf{R}}$ $\overline{\mathbf{U}}$, with $\overline{\mathbf{R}}$ denoting the macroscopic rotation tensor. In turn, the objectivity of $\widetilde{W}$ implies the macroscopic rotational balance equation $\overline{\mathbf{S}} \overline{\mathbf{F}}^{T}=\overline{\mathbf{F}} \overline{\mathbf{S}}^{T}$ (Hill, 1972). 
Noting that under the affine boundary condition implied by relation (6), the average or macroscopic deformation gradient is given by $\langle\mathbf{F}\rangle=\overline{\mathbf{F}}$, and correspondingly defining the average or macroscopic stress by $\overline{\mathbf{S}}=\langle\mathbf{S}\rangle$, the effective, or homogenized constitutive relation for the composite can be shown (Hill, 1972) to be given by

$$
\overline{\mathbf{S}}=\frac{\partial \widetilde{W}}{\partial \overline{\mathbf{F}}} .
$$

On the other hand, the effective elasticity tensor is defined by

$$
\widetilde{\mathbf{L}}(\overline{\mathbf{F}}) \equiv \frac{\partial^{2} \widetilde{W}}{\partial \overline{\mathbf{F}} \partial \overline{\mathbf{F}}}(\overline{\mathbf{F}}) .
$$

\subsection{Hypothesis on the constitutive behavior of the phases}

Recalling that $W$ cannot be assumed to be convex, in particular, because such an assumption would be inconsistent with the possible development of buckling and other types of bifurcation instabilities, as well as with the objectivity requirement, we list next some definitions that will be useful in the discussions below regarding appropriate constitutive assumptions for the materials and their stability (see, for example, Ball, 1977; Ciarlet, 1988; Dacorogna, 2007).

First, the function $W$ is said to be quasiconvex (Morrey, 1952) if

$$
\int_{D} W\left(\mathbf{X}_{0}, \mathbf{F}_{0}+\operatorname{Grad} \mathbf{x}^{\prime}\right) d V_{\mathbf{X}} \geq W\left(\mathbf{X}_{0}, \mathbf{F}_{0}\right) \times \operatorname{Vol}(D)
$$

for each fixed $\mathbf{X}_{0} \in \Omega_{0}$, for each constant second-order tensor $\mathbf{F}_{0}$, for each bounded subset $D$ and for all sufficiently smooth (e.g., Lipschitz continuous) deformation fluctuations $\mathbf{x}^{\prime}$ on $D$. Physically, it means that for any body made of the material at point $\mathbf{X}_{0}$, the minimum energy solution of any displacement boundary-value problem with zero body force and having affine deformation prescribed on its boundary is precisely that with homogeneous deformation gradient everywhere in the body. Thus, this condition excludes the possible formation of "domain microstructures" (i.e., minimizing sequences) with lower energies.

Second, letting $\mathbf{F}^{a} \equiv \operatorname{det}(\mathbf{F}) \mathbf{F}^{-T}$ denote the adjugate of the tensor $\mathbf{F}, W$ is said to be polyconvex if there is a function $f$ such that

$$
W\left(\mathbf{X}_{0}, \mathbf{F}\right)=f\left(\mathbf{X}_{0}, \mathbf{F}, \mathbf{F}^{a}, \operatorname{det} \mathbf{F}\right)
$$

with $f$ convex in each of its last three arguments, for fixed $\mathbf{X}_{0}$. 
Third, $W$ is rank-one convex at $\mathbf{F}_{0}$, if there exists a function $g\left(\mathbf{X}_{0}, t\right)=W\left(\mathbf{X}_{0}, \mathbf{F}_{0}+\right.$ $t \mathbf{m} \otimes \mathbf{N}$ ), which is convex in $t$ for all $\mathbf{m}$ and $\mathbf{N}$. When $W$ is sufficiently smooth, this condition is equivalent to the Legendre-Hadamard, or ellipticity condition

$$
m_{i} N_{j} L_{i j k l}\left(\mathbf{X}_{0}, \mathbf{F}_{0}\right) m_{k} N_{l} \geq 0, \quad \text { for all vectors } \mathbf{m}, \mathbf{N} \text {, and fixed } \mathbf{X}_{0}, \mathbf{F}_{0} .
$$

If $W$ is rank-one convex for all $\mathbf{F}_{0}$, then $W$ is said to be rank-one convex.

Moreover, if $W$ takes on finite values, we have the following result (Dacorogna, 2007) relating the above-defined generalized notions of convexity:

$W$ convex $\Longrightarrow W$ polyconvex $\Longrightarrow W$ quasiconvex $\Longrightarrow W$ rank-oneconvex.

For extended real-valued functions, polyconvexity implies both quasiconvexity and rank-one convexity, but quasiconvexity does not imply in general rank-one convexity. However, Conti (2008) has shown that quasiconvex energy functions incorporating incompressibility constraints are rank-one convex. It is further noted that quasiconvexity is a non-local condition, which is difficult to verify directly. On the other hand, polyconvexity and rank-one convexity can be verified locally for each value of $\mathbf{F}$, and in view of the implications (12), can be used to establish quasiconvexity.

Finally, it is also relevant to define strict rank-one convexity, or the strong ellipticity condition, by means of the same requirement (11) except that the inequality ' $\geq$ ' is replaced by a strict inequality ' $>$.' The physical meaning of strong ellipticity is that the material cannot admit solutions with discontinuous deformation gradients within a given phase (Truesdell and Noll, 1965; Ogden, 1997).

In this work, it will be assumed that $W$ is polyconvex and strongly elliptic, so that the homogeneous phases of the material cannot develop microstructures, nor shear-band-type localization instabilities. In particular, these assumptions (together with appropriate growth conditions) on $W$ would imply that if the material was made of only one homogeneous phase, the minimizing deformation in expression (5) would always be the uniform and unique deformation $\mathbf{x}=\overline{\mathbf{F}} \mathbf{X}$. However, as alluded to in the introduction, this is not necessarily the case for a heterogeneous material, and the stored-energy function of the composite may lose strong ellipticity, even when its phases are polyconvex and strongly elliptic. Thus, a hyperelastic composite material subjected to affine deformations on its boundary could in principle exhibit solutions that exhibit oscillations on length scales that are small compared to the size of the RVE, but still large relative to the size of the heterogeneities. In other words, the macroscopically uniform solution corresponding to the affine deformation applied on the boundary of the RVE may- 
after loss of strong ellipticity of the homogenized response-give way to solutions exhibiting "domain microstructures" (i.e., minimizing sequences tending to lower the energy of the energy). Therefore, as a consequence of the above-mentioned hypotheses on the properties of the constituent phases, if any bifurcation instabilities were to develop in expression (5) for a composite material, they would have to be due to the interaction of the different phases in the composite, and not to the intrinsic behavior of the constituent phases. In any case, because of the possible development of such macroscopic instabilities, it is necessary here to consider a more general definition of the macroscopic stored-energy function, which is able to account for the possible development of domain structures, after the onset of such instabilities.

\subsection{Generalized homogenization framework}

As already mentioned, for hyperelastic composites with periodic microstructures, more general definitions of the effective energy $\widetilde{W}$ have been given by Braides (1985) and Müller (1987). In particular, these more general definitions allow for the development of "microscopic" instabilities in the composite, where the principal solution, which is periodic on a single unit cell, gives way to periodic solutions on $N \times M \times L$ super-cells at sufficiently large deformations. Physically, this is due to the fact that solutions involving interactions between several unit cells may lead to lower effective energies. But the theory also predicts the possible development of "macroscopic" instabilities corresponding to long-wavelength instabilities which are not periodic at finite wavelengths, and can be determined by loss of strong ellipticity of the homogenized stored-energy function, as determined by the (one-cell periodic) principal solution (Geymonat et al., 1993).

On the other hand, for hyperelastic composites with random microstructures, the theory is less well developed. Clearly, macroscopic instabilities corresponding to loss of strong ellipticity of the homogenized stored-energy function can be easily computed and would still correspond to possible shear localization (long wavelength) instabilities (Lopez-Pamies and Ponte Castañeda, 2004, 2006b). However, due to the lack of periodicity, "microscopic" instabilities would not be expected to occur in composites with random microstructures. Indeed, recent work (Michel et al., 2007, 2010) suggests that the macroscopic instabilities may be the only relevant ones for random systems, since the microscopic instabilities in periodic systems tend to disappear as the periodicity of the microstructure is broken down (say, by perturbing randomly the positions of the pores or inclusions at the center of the unit cell). 
As a consequence, in this work, which as already stated will be focused on random composites, the following "heuristic" approach will be pursued. Assuming that the materials of interest have a stress-free configuration at $\overline{\mathbf{F}}=\mathbf{I}$, and that their behavior is characterized by the standard theory of linear elasticity for small enough deformations, it follows that, at least in a neighborhood of $\overline{\mathbf{F}}=\mathbf{I}$, the solution of the Euler-Lagrange equations associated with the variational problem (5) is unique, and gives the minimum energy. However, as the deformation progresses into the nonlinear regime, the composite material may reach a point at which this "principal" solution loses strong ellipticity and bifurcates into lower-energy solutions. To ensure that the effective stored-energy function for the hyperelastic composite includes such possible lower-energy "post-bifurcation" or "relaxed" solutions, we redefine $\widetilde{W}$ by replacing the min condition in Hill's original definition (5) of the effective stored-energy function by an inf, such that

$$
\widetilde{W}(\overline{\mathbf{F}})=\inf _{\mathbf{F} \in \mathscr{K}(\overline{\mathbf{F}})}\langle W(\mathbf{X}, \mathbf{F})\rangle
$$

where $\mathscr{K}(\overline{\mathbf{F}})$ is still given by expression (6), but the displacement fluctuations are required to belong to an appropriate functional space (where the infimum need not be attained). In this context, it should be noted that it follows from its definition (13) that the homogenized stored-energy $\widetilde{W}$ of the composite is quasiconvex, and therefore also rank-one convex (Geymonat et al., 1993). However, it need not be strictly rank-one convex, and the strong ellipticity condition, applied to the effective elasticity tensor $\widetilde{\mathbf{L}}$ of the composite, may be violated for some sufficiently large values of $\overline{\mathbf{F}}$.

In addition, it is also useful to define the "principal" estimate for the energy by means of the stationary variational statement:

$$
\widehat{W}(\overline{\mathbf{F}})=\operatorname{stat}_{\mathbf{F} \in \mathscr{K}(\overline{\mathbf{F}})}\langle W(\mathbf{X}, \mathbf{F})\rangle
$$

where it is emphasized that this energy is evaluated at the above-described "principal" solution of the pertinent Euler-Lagrange equations, starting from the unique solution in the neighborhood of the reference configuration with $\overline{\mathbf{F}}=\mathbf{I}$. In other words, we estimate the effective energy by means of expression (14) for $\widehat{W}$, starting from the unique solution for small deformations, but without worrying whether or not it actually gives the minimum energy for larger values of $\overline{\mathbf{F}}$. Then, in a second step, we estimate when such a solution ceases to be the minimum energy by determining the critical values of $\overline{\mathbf{F}}$ at which loss of strong ellipticity first occurs. These values correspond to the onset of macroscopic instabilities beyond which 
the physical relevance of the "principal" solution becomes questionable. Thus, from its definition, it is clear that $\widetilde{W}(\overline{\mathbf{F}})=\widehat{W}(\overline{\mathbf{F}})$ up to the onset of the first macroscopic instability. Beyond this point, $\widetilde{W}(\overline{\mathbf{F}}) \leq \widehat{W}(\overline{\mathbf{F}})$.

But how should we determine $\widetilde{W}$ beyond the possible onset of macroscopic instabilities? One possible answer would be the quasiconvexification $Q \widehat{W}$ of $\widehat{W}$. To formally argue that this is at least plausible, note that (Dacorogna, 2007)

$$
Q \widehat{W}(\overline{\mathbf{F}})=\inf _{\mathbf{u}(\mathbf{Y})}\left\langle\widehat{W}(\overline{\mathbf{F}}+\operatorname{Grad} \mathbf{u}(\mathbf{Y})\rangle_{V_{0}},\right.
$$

where the trial functions $\mathbf{u}$ vanish on the boundary and belong to some appropriate functional space (e.g., the set of Lipschitz-continuous functions) and the triangular brackets denote a volume average over some sufficiently large representative volume element $V_{0}$. As illustrated in Figure 1, after a macroscopic instability, $V_{0}$ breaks up into many "domains" $\Omega_{0}$, where the formula (14) for $\widehat{W}$ can still be used in each domain. In other words, the domains $\Omega_{0}$ include many fibers so that the separation of scales hypothesis is still satisfied within each $\Omega_{0}$, and the response of the domain can still be estimated by the principal solution.

Now, making use of the definition (14) for $\widehat{W}$ in expression (15), we find that

$$
Q \widehat{W}(\overline{\mathbf{F}})=\inf _{\mathbf{u}(\mathbf{Y})}\left\langle\underset{\mathbf{u}^{\prime}(\mathbf{X})}{\operatorname{stat}}\left\langle W\left(\mathbf{X}, \overline{\mathbf{F}}+\operatorname{Grad} \mathbf{u}^{\prime}(\mathbf{X})+\operatorname{Grad} \mathbf{u}(\mathbf{Y})\right)\right\rangle_{\Omega_{0}}\right\rangle_{V_{0}},
$$

where it has been implicitly assumed that the scale of variation of $\mathbf{u}$ in $V_{0}$ is larger than that of $\mathbf{u}^{\prime}$ in $\Omega_{0}$. Now, since the class of stationary solutions in the definition of (14) for $\widehat{W}$ is included in the class of solutions leading to the infimum, it also follows that

$$
Q \widehat{W}(\overline{\mathbf{F}}) \geq \inf _{\mathbf{u}(\mathbf{Y})}\left\langle\inf _{\mathbf{u}^{\prime}(\mathbf{X})}\left\langle W\left(\mathbf{X}, \overline{\mathbf{F}}+\operatorname{Grad} \mathbf{u}^{\prime}(\mathbf{X})+\operatorname{Grad} \mathbf{u}(\mathbf{Y})\right)\right\rangle_{\Omega_{0}}\right\rangle_{V_{0}}
$$

On the other hand, since the domain structure depicted in Figure 1(c) is but one possible solution to the infimum problem in the definition (13) of the homogenized energy $\widetilde{W}$, it follows that

$$
\inf _{\mathbf{u}(\mathbf{Y})}\left\langle\inf _{\mathbf{u}^{\prime}(\mathbf{X})}\left\langle W\left(\mathbf{X}, \overline{\mathbf{F}}+\operatorname{Grad} \mathbf{u}^{\prime}(\mathbf{X})+\operatorname{Grad} \mathbf{u}(\mathbf{Y})\right)\right\rangle_{\Omega_{0}}\right\rangle_{V_{0}} \geq \inf _{\mathbf{u}(\mathbf{Y})}\langle W(\mathbf{Y}, \overline{\mathbf{F}}+\operatorname{Grad} \mathbf{u}(\mathbf{Y}))\rangle_{V_{0}} .
$$

In conclusion, we have (formally) argued that

$$
\widetilde{W}(\overline{\mathbf{F}}) \leq Q \widehat{W}(\overline{\mathbf{F}}) .
$$




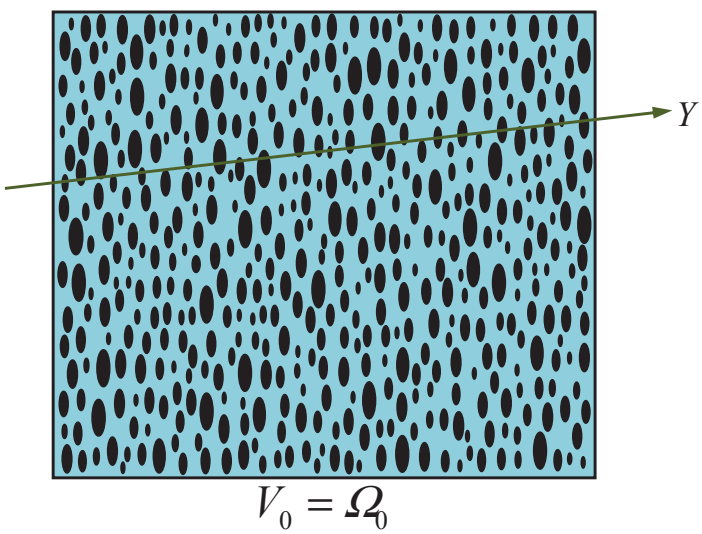

(a)

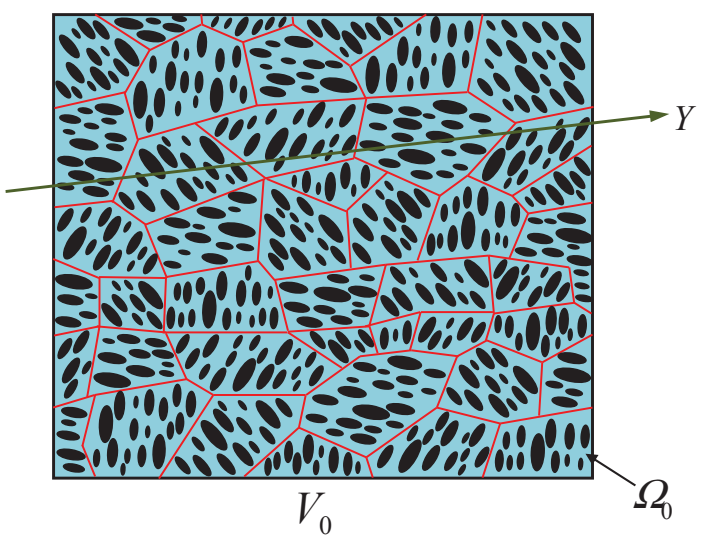

(c)

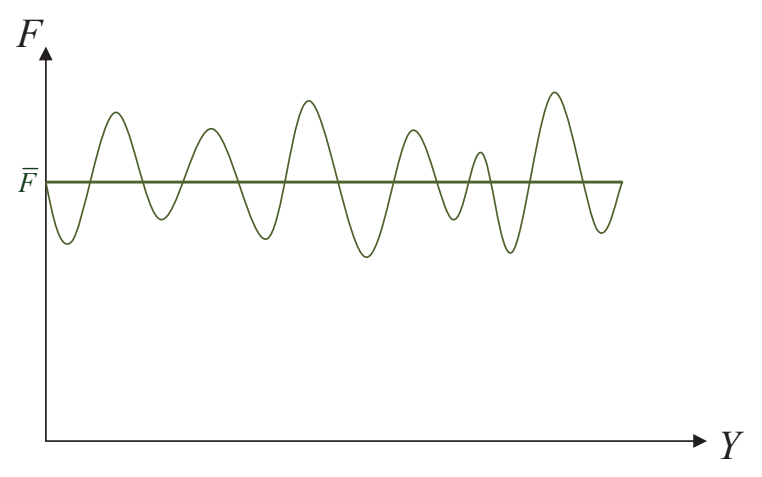

(b)

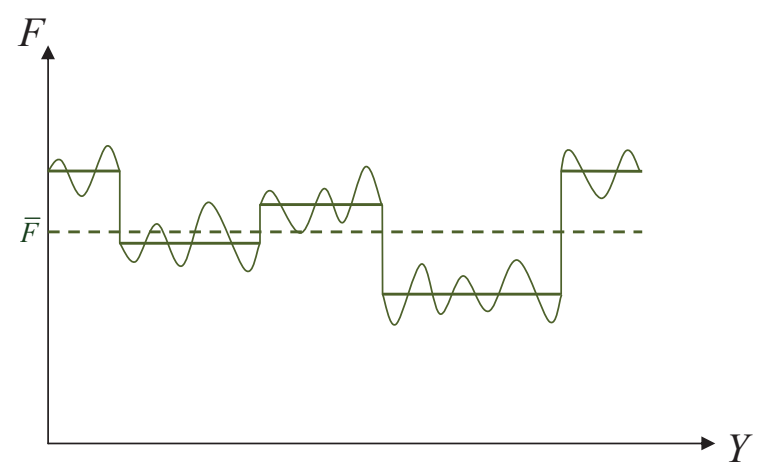

(d)

Figure 1: Schematic representation of the possible development of domain structures in a hyperelastic composite. As depicted in (a) and (b), prior to the development of macroscopic instabilities, the RVE $V_{0}=\Omega_{0}$ deforms in a statistically uniform fashion, and the microscopic deformation gradient fields oscillate about a macroscopically uniform deformation gradient that is compatible with the affine displacement conditions that are applied on the boundary of the RVE. The energy associated with this solution is called the "principal" or "mesoscopic" energy $\widehat{W}$. As depicted in (c) and (d), beyond the onset of a macroscopic instability, the the RVE $V_{0}$ breaks up into domains $\Omega_{0}$, which are regions containing many inhomogeneities, where the microscopic fields oscillate about a uniform field on the "mesoscopic" scale of the domains. However, the mesoscopically uniform fields in the domains vary from domain to domain (and may even vary within the domains on a scale that is still large compared to the heterogeneities) in such a way that the overall deformation is compatible with the affine overall deformation on the boundary. The energy associated with this solution is called the "relaxed" or "macroscopic" energy $\widetilde{W}$. Note that $\widetilde{W}=Q \widehat{W} \leq \widehat{W}$, since the energy of the domains can still be estimated from $\widehat{W}$. 
This result also follows by taking the quasiconvex envelope of the inequality $\widetilde{W} \leq \widehat{W}$, and noting that $\widetilde{W}$ is quasiconvex. However, the more detailed arguments presented above suggest that strict equality would hold in the inequality (19) provided that two conditions be satisfied. First, equality should hold in (17). This condition would be satisfied if the infimum over $\mathbf{u}^{\prime}$ is actually the stationary solution in expression (16), which means that the average deformation gradient over the "grains" in Figure 1 is small enough so that $\widetilde{W}=\widehat{W}$ still holds in the grains. (Otherwise, the grains would have to once again break up into subgrains.) Second, equality should also be required in (18). In turn, this condition would be satisfied if the typical size of the grains is large compared to the fiber size, so that the "iterated" homogenization on the left of this inequality would be identical to the more general (one-shot) homogenization result on the right.

For the hyperelastic composites of interest here, the first condition can be easily checked a posteriori, while the second condition would also be expected to hold due to the randomness of the relevant microstructures, which should preclude the formation of "microscopic" instabilities involving a finite (small) number of heterogeneities (akin to the microscopic instabilities in periodic composites). Thus, in this work, we will assume that both of these conditions hold, and will estimate the homogenized stored-energy function $\widetilde{W}$ of the composites directly from the quasiconvexification of the "principal" solution $\widehat{W}$ for the energy, so that

$$
\widetilde{W}(\overline{\mathbf{F}})=Q \widehat{W}(\overline{\mathbf{F}}) .
$$

In this connection, it should be noted that the second condition concerning the separation of scales assumption has also been made implicitly in the work of DeSimone and Dolzmann (2000, 2002), where the "mesoscopic" stored-energy function of the nematic liquid crystal elastomer is taken from the "homogenized" result of Blandon et al. (1993), which has been obtained from the molecular theory of rubber elasticity. In this sense, the stored-energy function $\widehat{W}$, as given by (14), could also be thought of as a "mesoscopic" energy, while $\widetilde{W}$, as given by (13), would be the true "macroscopic" or relaxed energy.

In the rest of this paper, we consider the implementation of this generalized framework for estimating the relaxed energy of hyperelastic composites in the context of a specific example. Thus, we will work with the two-dimensional reinforced elastomers of Lopez-Pamies and Ponte Castañeda (2006b), for which an estimate was obtained for the "principal" or "mesoscopic" effective stored-energy function $\widehat{W}$, as defined by expression (14). This estimate was found to lose strong ellipticity when sufficient compression was applied along the long axes of the 

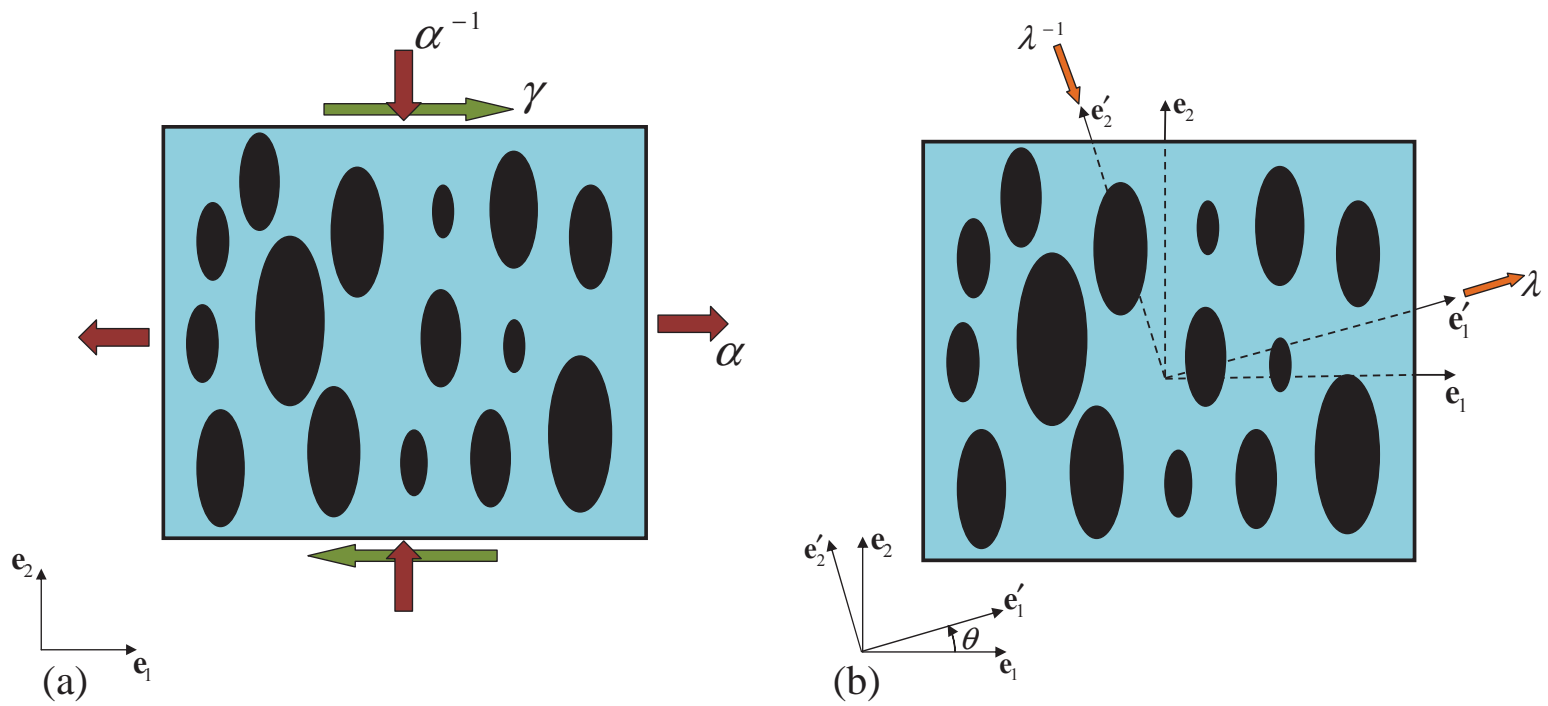

Figure 2: Schematic illustration of the microstructure in the fiber-reinforced composite under plane strain loading. Parts (a) and (b) show two equivalent ways (modulo a rigid body rotation) to deform the material.

fibers. In the remaining sections, we construct the rank-one convexification of this energy function and show that it is polyconvex, from which it will be deduced that it must correspond to the quasiconvexification of the energy, and therefore, to the relaxed energy $\widetilde{W}$ of the composite, as given by expressions (13) and (20).

\section{Plane strain loading of fiber-reinforced elastomers}

\subsection{Microstructural and loading variables}

In this section, we consider plane strain deformations (in the 1-2 plane) of fiber-reinforced elastomers, such that the (rigid) cylindrical fibers are perpendicular to the plane of the deformation (i.e., the fibers are aligned in the 3 direction). Figure 2 shows a schematic representation of the transverse cross section of the microstructure in the undeformed configuration relative to the fixed laboratory frame defined by the Cartesian triad $\left\{\mathbf{e}_{i}\right\}(i=1,2,3)$. The fibers are assumed to have initial volume fraction $c_{0}$, and to have elliptical cross section with the same aspect ratio $w$. In addition, they are assumed to be aligned (in the plane of the deformation), such that the short axis of the ellipses defines the (in-plane) fiber direction $\mathbf{N}_{f}=\mathbf{e}_{1}$. On account of the two-dimensional character of the problem, we 
can further assume without loss of generality that $w \geq 1$, corresponding to fibers with long axes aligned with the $\mathbf{e}_{2}$ direction.

The constitutive behavior of the elastomeric matrix phase is characterized by the stored-energy function $W^{(1)}$, which is assumed to be an objective and isotropic function of the deformation gradient tensor $\mathbf{F}$. As a consequence, $W^{(1)}$ can be written as a general function of the isotropic invariants of the right Cauchy-Green deformation tensor $\mathbf{C}=\mathbf{F}^{T} \mathbf{F}$. For plane deformations, the relevant principal invariants are $I=\operatorname{tr}(\mathbf{C})$ and $J=\operatorname{det} \mathbf{F}=\sqrt{\operatorname{det}(\mathbf{C})}$, and, in this work we therefore restrict our attention to stored-energy functions of the generalized neo-Hookean type, such that

$$
W^{(1)}(\mathbf{F})=g(I)+h(J)+\frac{1}{2} \mu^{\prime(1)}(J-1)^{2},
$$

where $g(I)$ and $h(J)$ are assumed to be twice continuously differentiable functions, satisfying the conditions: $g(2)=h(1)=0, g^{\prime}(2)=\mu^{(1)} / 2, h^{\prime}(1)=-\mu^{(1)}$, and $4 g^{\prime \prime}(2)+h^{\prime \prime}(1)=\mu^{(1)}$. In these expressions, the primes on the functions $g$ and $h$ stand for derivatives with respect to the appropriate variables, and $\mu^{(1)}$ and $\mu^{\prime(1)}$ denote the Lamé moduli of the isotropic matrix in the ground state. In addition, the functions $g$ and $h$ are assumed to be such that $W^{(1)}$ is polyconvex and strictly rank-one convex (strongly elliptic).

A well-known example of the general form (21), which captures the limiting chain extensibility of elastomers, is the model of Gent (1996), defined by

$$
W^{(1)}(\mathbf{F})=-\frac{J_{m} \mu^{(1)}}{2} \ln \left(1-\frac{I-2}{J_{m}}\right)-\mu^{(1)} \ln (J)+\frac{1}{2}\left(\mu^{\prime(1)}-2 \frac{\mu^{(1)}}{J_{m}}\right)(J-1)^{2},
$$

for $0<I<2+J_{m}$ and $J>0$, and infinity otherwise. In this expression, the dimensionless parameter $J_{m}$ is the strain-locking parameter, such that the energy function and associated stresses blow up as $I \rightarrow 2+J_{m}$. It should be remarked that the Gent model (22) is polyconvex and strongly elliptic for all deformations, provided that the conditions $\mu^{(1)}>0, J_{m}>0, \mu^{\prime(1)}>2 \mu^{(1)} / J_{m}$ be satisfied. Note also that the Gent model (22) reduces to the compressible neo-Hookean model, which never locks up, in the limit as $J_{m} \rightarrow \infty$. In turn, this model reduced to the classical incompressible neo-Hookean model in the limit as $\mu^{\prime(1)} \rightarrow \infty$.

Focusing our attention on the two-dimensional problem defined by the abovedescribed fiber-reinforced composites subjected to plane strain deformations, the effective stored-energy function $\widetilde{W}$ (or $\widehat{W}$ ) characterizing the macroscopic behavior of the composite can be expressed in terms of an appropriate set of invariants of the deformation gradient. (Henceforth, for simplicity in notation, we drop the 
bar from macroscopic deformation and stress variables such as $\overline{\mathbf{F}}$ and $\overline{\mathbf{S}}$ in (7).) These invariants are functions of $\mathbf{C}=\mathbf{F}^{T} \mathbf{F}$ and of the preferred (fiber) direction $\mathbf{N}_{f}$. One possible set of invariants (Spencer, 1984) is given by

$$
I_{1}=\operatorname{tr}(\mathbf{C}), \quad I_{2}=\mathbf{N}_{f} \cdot \mathbf{C} \mathbf{N}_{f}, \quad J=\operatorname{det}(\mathbf{F})=\sqrt{\operatorname{det}(\mathbf{C})} .
$$

However, the choice of the set of invariants is not unique, and in this work, it proves useful to introduce alternative sets of invariants, as shown next.

Writing the Cartesian components (with respect to the laboratory coordinates defined by the $\left\{\mathbf{e}_{i}\right\}$ ) of the applied deformation $\mathbf{F}$ in matrix form as

$$
F_{i j}=\left(\begin{array}{ll}
F_{11} & F_{12} \\
F_{21} & F_{22}
\end{array}\right)
$$

where it is recalled that $J=F_{11} F_{22}-F_{12} F_{21}>0$, it can be shown that such a general deformation gradient $\mathbf{F}$ can be also expressed as the product

$$
\mathbf{F}=\mathbf{R}_{0} \mathbf{F}_{0}
$$

where the Cartesian components of $\mathbf{F}_{0}$ are given by

$$
\left(F_{0}\right)_{i j}=\sqrt{J}\left(\begin{array}{cc}
\alpha & \gamma \\
0 & \alpha^{-1}
\end{array}\right)
$$

and $\mathbf{R}_{0}$ is a proper orthogonal tensor with corresponding components given by

$$
\left(R_{0}\right)_{i j}=\left(\begin{array}{cc}
\cos \psi_{0} & -\sin \psi_{0} \\
\sin \psi_{0} & \cos \psi_{0}
\end{array}\right) .
$$

In expression (26), $\alpha$ and $\gamma$ are given by

$$
\gamma= \begin{cases}\alpha & =\sqrt{\frac{I_{2}}{J}}, \\ \sqrt{\frac{I_{1}}{J}-\frac{J}{I_{2}}-\frac{I_{2}}{J}}, & \text { if } \quad F_{11} F_{12}+F_{21} F_{22} \geq 0, \\ -\sqrt{\frac{I_{1}}{J}-\frac{J}{I_{2}}-\frac{I_{2}}{J}}, & \text { if } \quad F_{11} F_{12}+F_{21} F_{22} \leq 0,\end{cases}
$$

while the angle $\psi_{0}$ in (27) is determined by the following equation

$$
\frac{\left(F_{22}-F_{11}\right) \sin \psi_{0}+\left(F_{21}+F_{12}\right) \cos \psi_{0}}{\left(F_{11}+F_{22}\right) \cos \psi_{0}+\left(F_{21}-F_{12}\right) \sin \psi_{0}}=\frac{\gamma \alpha}{\alpha^{2}+1} .
$$


Note that $I_{1} \geq I_{2}+J^{2} / I_{2}$ and $I_{2}>0$.

Next, recalling the objectivity of the effective stored-energy function $\widetilde{W}$ (or $\widehat{W}$ ), it follows that it suffices to consider deformation gradients of the form (26), and therefore the variables $\alpha, \gamma$ and $J$ constitute an equivalent set of invariants (to $I_{1}, I_{2}$ and $J$ ). For ease of notation, we therefore re-label the macroscopic deformation gradient (26) as $\mathbf{F}$ (i.e., we drop the subscript 0 ). In addition, it is noted that the components of $\mathbf{F}$ further reduce to

$$
F_{i j}=\left(\begin{array}{cc}
\alpha & \gamma \\
0 & \alpha^{-1}
\end{array}\right)
$$

for isochoric deformations (when $J=\operatorname{det}(\mathbf{F})=1$ ). It is further noted that this deformation state corresponds to a superposition of a pure shear state with stretches $\alpha$ and $1 / \alpha(0<\alpha<\infty)$ along the $\mathbf{e}_{1}$ and $\mathbf{e}_{2}$ directions, respectively, together with a transverse shear of magnitude $\gamma(-\infty<\gamma<\infty)$ along the $\mathbf{e}_{1}$ direction. For clarity, the loading parameters $\alpha$ and $\gamma$ are shown schematically in Figure 2(a).

In turn, the deformation gradient $\mathbf{F}$, defined by (31), may be rewritten in the form

$$
\mathbf{F}=\mathbf{R} \mathbf{U}=\mathbf{R} \mathbf{Q} \mathbf{D} \mathbf{Q}^{T},
$$

where $\mathbf{R}$ and $\mathbf{U}$ correspond, respectively, to the rotation and right-stretch tensors associated with the polar decomposition of $\mathbf{F}$ in (31), and $\mathbf{Q}$ and $\mathbf{D}$ correspond to a proper orthogonal and diagonal tensor, respectively. Relative to the laboratory coordinates, the tensors $\mathbf{R}, \mathbf{Q}$ and $\mathbf{D}$ have matrix representations

$R_{i j}=\left(\begin{array}{cc}\cos \psi & -\sin \psi \\ \sin \psi & \cos \psi\end{array}\right), \quad Q_{i j}=\left(\begin{array}{cc}\cos \theta & -\sin \theta \\ \sin \theta & \cos \theta\end{array}\right), \quad D_{i j}=\left(\begin{array}{cc}\lambda & 0 \\ 0 & 1 / \lambda\end{array}\right)$,

where $\lambda$ and $1 / \lambda$ are the principal stretches of the deformation, and $\theta$ denotes the angle (positive anticlockwise) of the in-plane Lagrangian axes relative to the laboratory axes. In addition, the angle $\psi$ is the rigid-body rotation associated with the deformation F. Since, once again, the rigid-body rotation does not affect the constitutive response, we can ignore it, and consider the response of the material under the pure stretch $\mathbf{U}$. Thus, Figure 2(b) depicts a pure shear deformation of magnitude $\lambda$ at the "loading angle" $\theta$.

Without loss of generality, we can assume that $\lambda \geq 1$, provided that we take $-\pi / 2 \leq \theta \leq \pi / 2$ in (33). Then, the principal stretch $\lambda$ and loading angle $\theta$ can be related to the loading variables $\alpha$ and $\gamma$ in (31) via the expressions

$$
\lambda=\frac{1}{\sqrt{2} \alpha} \sqrt{\gamma^{2} \alpha^{2}+\alpha^{4}+1+s}
$$


and

$$
\theta=\arctan \left(\frac{1}{2} \frac{\gamma^{2} \alpha^{2}-\alpha^{4}+1+s}{\gamma \alpha^{3}}\right)
$$

where

$$
s=\sqrt{\left[\gamma^{2} \alpha^{2}+\left(\alpha^{2}-1\right)^{2}\right]\left[\gamma^{2} \alpha^{2}+\left(\alpha^{2}+1\right)^{2}\right]} .
$$

In addition, the rigid-body rotation $\psi$ can be obtained in terms of $\alpha$ and $\gamma$ via

$$
\psi=-\arcsin \left\{\frac{\left(\lambda^{2}-1\right) \sin (\theta) \cos (\theta)}{\lambda \alpha}\right\},
$$

where $\lambda$ and $\theta$ are given by expressions (34) and (35), respectively.

\subsection{Principal, or mesoscopic energy}

For the class of fiber-reinforced elastomers defined in the previous subsection, Lopez-Pamies and Ponte Castañeda (2006a; 2006b) derived a closed-form expression for the effective stored-energy $\widehat{W}(\mathbf{F})$, as defined by expression (14), characterizing the principal solution of the macroscopic response (i.e., the response prior to the onset of instabilities or bifurcated paths). For this purpose, use was made of an appropriate adaptation of the generalized secant second-order homogenization method initially developed for plastic composites (Ponte Castañeda, 2002), by considering rigid fibers and taking the incompressible limit $\left(\mu^{\prime(1)} \rightarrow \infty\right)$ of the matrix stored-energy function (21). The resulting energy function was found to satisfy the overall incompressibility constraint $(J=\operatorname{det}(\mathbf{F})=1)$ and can be written in the form

$$
\widehat{W}(\mathbf{F})=\widehat{\Phi}(\lambda, \theta)+K(\operatorname{det} \mathbf{F})
$$

where

$$
K(J)=\left\{\begin{array}{l}
0, \quad \text { if } \quad J=1 \\
+\infty, \text { otherwise }
\end{array}\right.
$$

and

$$
\widehat{\Phi}(\lambda, \theta)=(1-c) g(\hat{I})
$$

In this last expression, $\lambda$ and $\theta$ are defined by expressions (32) and (33), respectively, and $\hat{I}$ is given by

$$
\begin{aligned}
\hat{I} & =\frac{1}{(1-c)^{2} \lambda^{2} w}\left\{c\left(1+\lambda^{2}\right)^{2}+\left[1+2 c(c-2) \lambda^{2}+\lambda^{4}\right] w+c w^{2}\left(1+\lambda^{2}\right)^{2}\right. \\
& \left.-c\left(\lambda^{4}-1\right)\left(w^{2}-1\right) \sin (\varphi) \sin (\varphi-2 \theta)-2 c \lambda\left(1+\lambda^{2}\right)\left(1+w^{2}\right) \cos (\varphi)\right\} .
\end{aligned}
$$


where $c=c_{0}^{(2)}$ denotes the volume fraction of rigid fibers (note that the fibers volume fraction remains the same in the deformed configuration because of the matrix incompressibility), and $w$ is the aspect ratio of the elliptical cross-section of the fibers. In addition, consistent with macroscopic objectivity, the angle $\varphi=$ $\phi-\psi$ is the relative rotation of the particles (i.e., the average rotation angle of the fibers minus the macroscopic rotation of the homogenized material as determined by the first expression in (33)). This angle is measured relative to the laboratory axes (+ anticlockwise), and is determined as a function of the loading parameters $\lambda$ and $\theta$, for a given value of the fiber aspect ratio $w$, by means of the kinematical equation

$$
2 \lambda\left(w^{2}+1\right) \sin (\varphi)-\left(\lambda^{2}-1\right)\left(w^{2}-1\right) \sin [2(\varphi-\theta)]=0 .
$$

For completeness, it is noted that the solution for the above equation (41) can be written in the form

$$
\varphi=\arctan \left(\frac{2 b z-\sin \theta}{\cos \theta}\right)+\theta,
$$

where $b=\left(\lambda^{2}-1\right)\left(w^{2}-1\right) /\left[2 \lambda\left(w^{2}+1\right)\right]$, and $z$ is a real root of the fourth-order polynomial equation

$$
4 b^{2} z^{4}-4 \sin (\theta) b z^{3}+\left(1-4 b^{2}\right) z^{2}+4 \sin (\theta) b z-\sin ^{2}(\theta)=0 .
$$

(In fact, $z$ is the negative real root for $0<\theta<\pi / 2$, and the positive real root for $-\pi / 2<\theta<0$.)

While the use of the loading variables $\lambda$ and $\theta$ was helpful in the derivation of the result (38) for $\widehat{W}(\mathbf{F})$, these variables are not the most convenient in terms of visualizing the possible instabilities that may develop for a given loading path in the space of deformation gradients. In this sense, it is more useful to describe the energy function in terms of the loading variables $\alpha$ and $\gamma$, and we therefore introduce the energy function $\widehat{\Psi}(\alpha, \gamma)$, obtained from $\widehat{\Phi}(\lambda, \theta)$ by substituting the expressions (34) and (35) for $\lambda$ and $\theta$ in terms of $\alpha$ and $\gamma$ into expression (40) for $\widehat{\Phi}(\lambda, \theta)$, such that

$$
\widehat{\Psi}(\alpha, \gamma)=\widehat{\Phi}(\lambda, \theta)
$$

The resulting function $\widehat{\Psi}$ can be given an explicit expression in terms of $\alpha$ and $\gamma$, but it is too lengthy to be included here. However, for later reference, it is useful to remark that the function $\widehat{\Psi}(\alpha, \gamma)$ is symmetric about $\gamma=0$, i.e.,

$$
\widehat{\Psi}(\alpha,-\gamma)=\widehat{\Psi}(\alpha, \gamma)
$$


as one would expect from the symmetry of the loading with respect to the microstructure.

The macroscopic stress tensor associated with the incompressible strain energy (40) may be obtained via expression (7) by appropriately accounting for the incompressibility constraint. In terms of the macroscopic Cauchy stress, we have that

$$
\mathbf{T}=\frac{\partial \widehat{W}}{\partial \mathbf{F}} \mathbf{F}^{T}-p \mathbf{I}
$$

where $p$ is an arbitrary hydrostatic pressure. For plane deformations, it is useful to consider the normal stress difference $T_{11}-T_{22}$ and the shear stress $T_{12}$, where the components refer to the laboratory coordinates (cf., Figure 2(a)) and correspond to the axial stretch (perpendicular to the long axis of the fibers) $\alpha$ and the shear strain (transverse to the long axis of the fibers) $\gamma$. They are independent of the hydrostatic pressure and are most simply determined in terms of $\lambda$ and $\theta$ via the expressions

$$
\begin{gathered}
T_{11}-T_{22}=\frac{1}{\lambda^{4}-1}\left[\lambda\left(\lambda^{4}-1\right) \cos (2 \theta+2 \psi) \frac{\partial \widehat{\Phi}}{\partial \lambda}-2 \lambda^{2} \sin (2 \theta+2 \psi) \frac{\partial \widehat{\Phi}}{\partial \theta}\right] \\
T_{12}=\frac{1}{2\left(\lambda^{4}-1\right)}\left[\lambda\left(\lambda^{4}-1\right) \sin (2 \theta+2 \psi) \frac{\partial \widehat{\Phi}}{\partial \lambda}+2 \lambda^{2} \cos (2 \theta+2 \psi) \frac{\partial \widehat{\Phi}}{\partial \theta}\right]
\end{gathered}
$$

where the derivatives $\partial \widehat{\Phi} / \partial \lambda$ and $\partial \widehat{\Phi} / \partial \theta$ are given by expressions (A.1) in Appendix A. Similarly, appropriate (bounded) traces for the components of the effective tangent modulus or elasticity tensor

$$
\widehat{\mathbf{L}}(\mathbf{F}) \equiv \frac{\partial^{2} \widehat{W}}{\partial \mathbf{F} \partial \mathbf{F}}(\mathbf{F})
$$

are obtained in Appendix B in terms of the pertinent derivatives of $\widehat{\Phi}$ to check the strong ellipticity condition.

\subsection{Numerical examples of the principal solution}

In this subsection, we present representative examples of the principal solution for the macroscopic response of the fiber-reinforced elastomers described in the previous subsections undergoing plane deformations of the form (31). For simplicity, results for the energy $\widehat{\Psi}$ and the associated stress measures are normalized by the ground-state shear modulus (that is, $\mu^{(1)}=1$ ). In particular, we are 


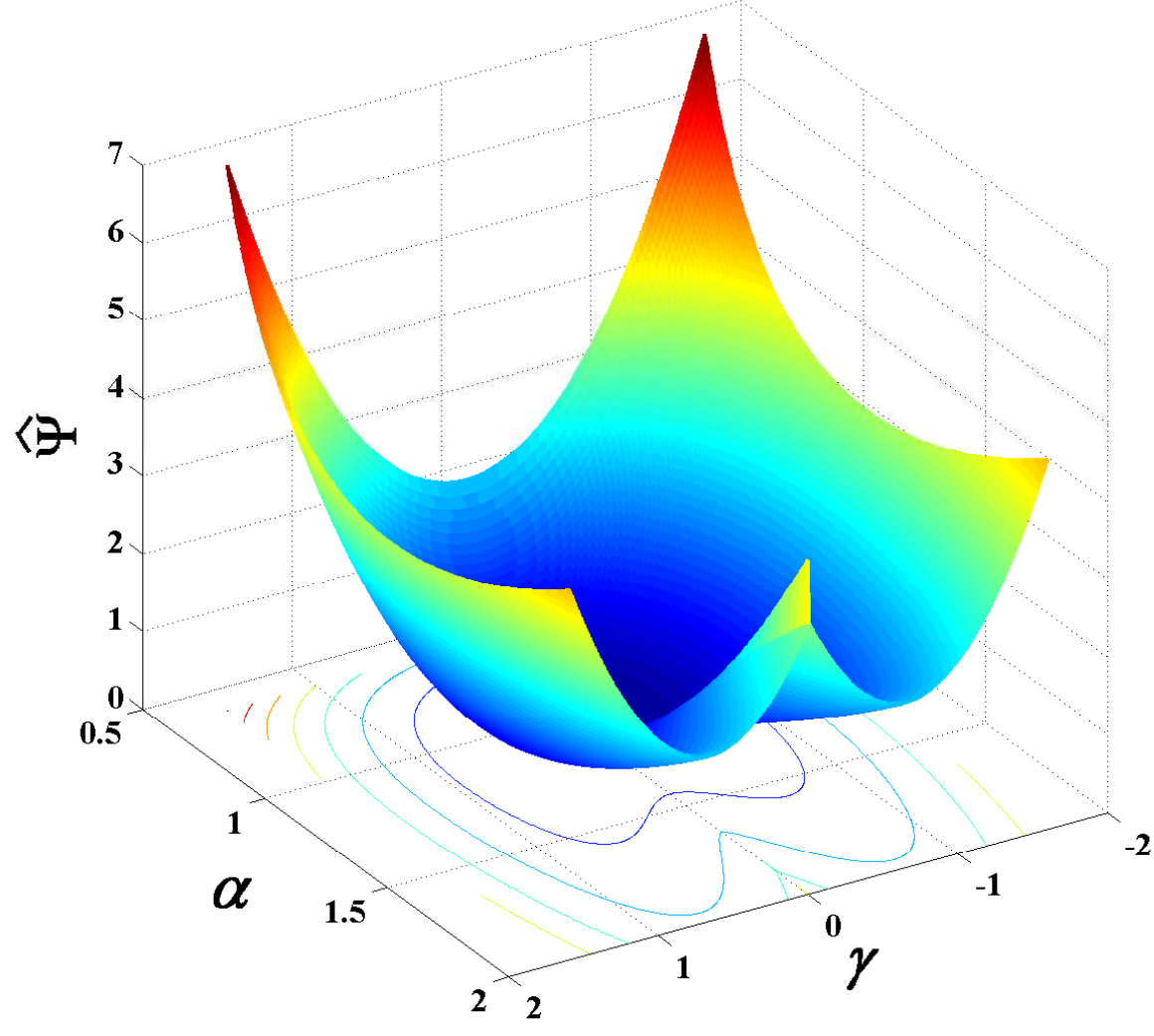

Figure 3: Principal estimate for the macroscopic stored-energy function of fiber-reinforced, neoHookean elastomers with fiber volume fraction $c=0.3$ and fiber aspect ratio $w=10$, as a function of loading variables $\alpha$ and $\gamma$, as depicted in Fig. 2(a).

interested in the loss of convexity of $\widehat{\Psi}$, which as we will see has implications for the stability and possible development of bifurcated solutions, as well as in the evolution of the microstructure, which is at the root of such instabilities.

Figure 3 shows a plot of the (principal solution for the) stored-energy function $\widehat{\Psi}$, defined by (40) and (44), of the neo-Hookean fiber-reinforced elastomer, as a function of the deformation variables $\alpha$ and $\gamma$, for fixed values of the fiber volume fraction $(c=0.3)$ and fiber aspect ratio $(w=10)$. It is recalled that $\alpha>1$ corresponds to a pure shear deformation with tension perpendicular to the long axis of the fibers (and compression along the long axis of the fibers), while $\gamma \neq 0$ corresponds to shear transverse to the long axis of the fibers. We observe from 
this figure that the energy landscape is symmetric with respect to the line $\gamma=0$, and is an increasing function of $\alpha(1 / \alpha)$ when $\alpha>1(\alpha<1)$. We further observe from this figure that, when $\alpha>1$, the energy develops a "crease" centered about the plane $\gamma=0$, and it becomes a decreasing function of $\gamma($ for $\gamma>0$ ) inside the crease region.

It was remarked by Lopez-Pamies and Ponte Castañeda (2006b) that the fiberreinforced elastomer loses strong ellipticity (SE) for "aligned" loadings $(\gamma=0)$ at some critical stretch $\alpha=\alpha_{c r}$, when the incremental shear modulus transverse to the long fiber axis vanishes (i.e., when $L_{1212}=0$ ). This point corresponds precisely to the 'tip' of the crease, where the energy function just loses convexity. Thus, the crease region may be identified with loss of global convexity of the function $\widehat{\Psi}(\alpha, \gamma)$, and can be described by the interval $\left[-\gamma_{c r}^{C}, \gamma_{c r}^{C}\right]$, where $\gamma_{c r}^{C}$ depends on $\alpha$, starting from zero at $\alpha=\alpha_{c r}$. Note that for fixed values of $\alpha$ with $\alpha>\alpha_{c r}$, the two values $\gamma= \pm \gamma_{c r}^{C}$ correspond to the two minima of the function $\widehat{\Psi}(\alpha, \gamma)$. As a consequence, the critical values of $\gamma$ may be determined from the condition $\partial \widehat{\Psi}(\alpha, \gamma) / \partial \gamma=0$, or equivalently, from the condition $S_{12}=T_{12}=0$, where $T_{12}$ is given by expression (48). On the other hand, the energy remains convex and an increasing function of $\gamma$ outside of the crease region.

Figure 4 presents plots of the principal solution for the energy and the associated particle rotation and stresses for a fiber-reinforced neo-Hookean elastomer with $c=0.3$ and $w=10$, as functions of $\gamma$, for several fixed values of $\alpha$. In these plots, the bullet ' $\bullet$ ' and diamond ' $'$ ' markers denote the points at which the energy function loses (local) strong ellipticity and global convexity (with respect to the variables $\alpha$ and $\gamma$ ), respectively. More specifically, Figs. 4(a) and (b) show results for the stored-energy function $\widehat{\Psi}$ and (in-plane) rotation of fibers, respectively. We observe from Figure 4(a) that the energy is convex in $\gamma$ for $\alpha<1$, but it loses (global) convexity, as well as strong ellipticity, for values of $\alpha>\alpha_{c r}$ (in this case, $\alpha_{c r} \approx 1.14$ ). Interestingly, the energy develops two wells that are symmetrically disposed about $\gamma=0$, and is therefore non-convex between the bottoms of these wells (which are labelled by the diamond markers). The loss of (local) strong ellipticity of the energy (labelled by circular markers) takes place inside the non-convex region. We further observe from Figure 4(a) that the energy exhibits a sharp peak at $\gamma=0$ for sufficiently large values of $\alpha$. The development of the non-convex region can be understood by making contact with the corresponding results for rotation of fibers shown in Figure 4(b). Thus, as can be seen in this figure, the rate of rotation of the particles increases dramatically with $\alpha$ near $\gamma=0$. This means that for fixed $\alpha$, the application of a small transverse shear $\gamma$ results in larger and larger amounts of stress being released as a consequence 

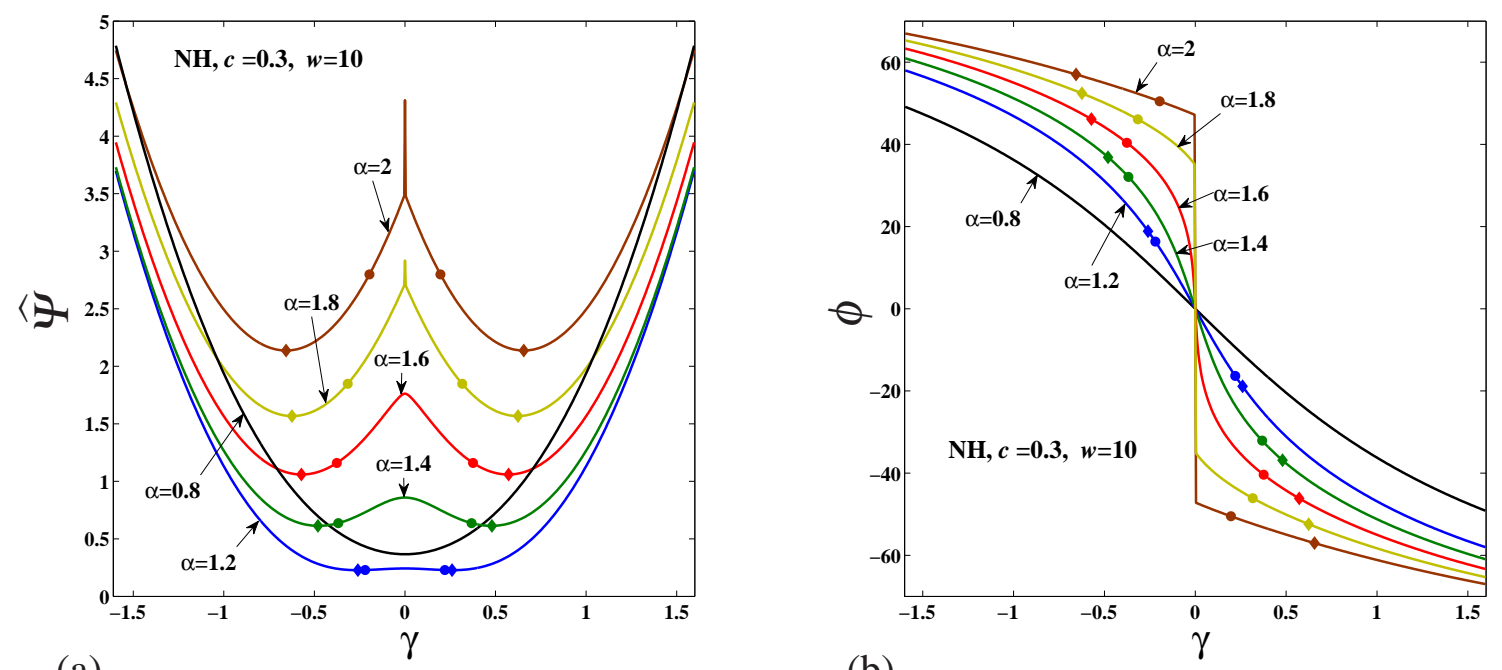

(a)

(b)

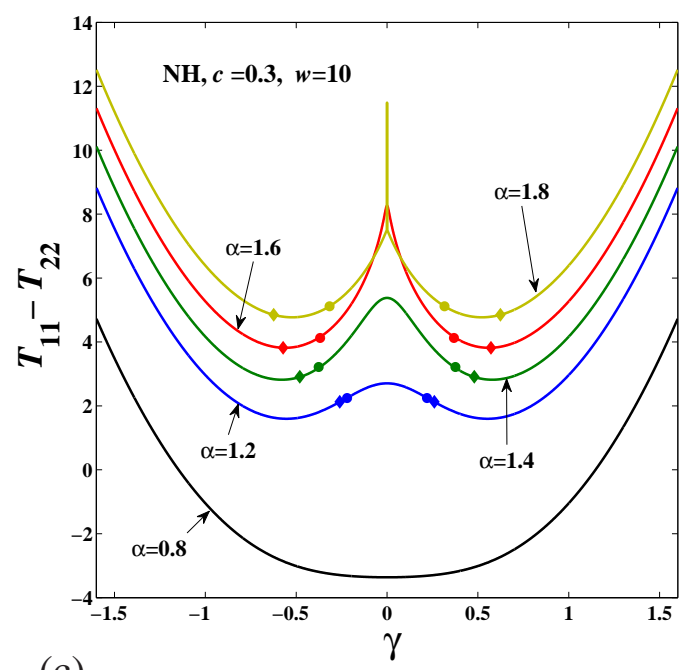

(c)

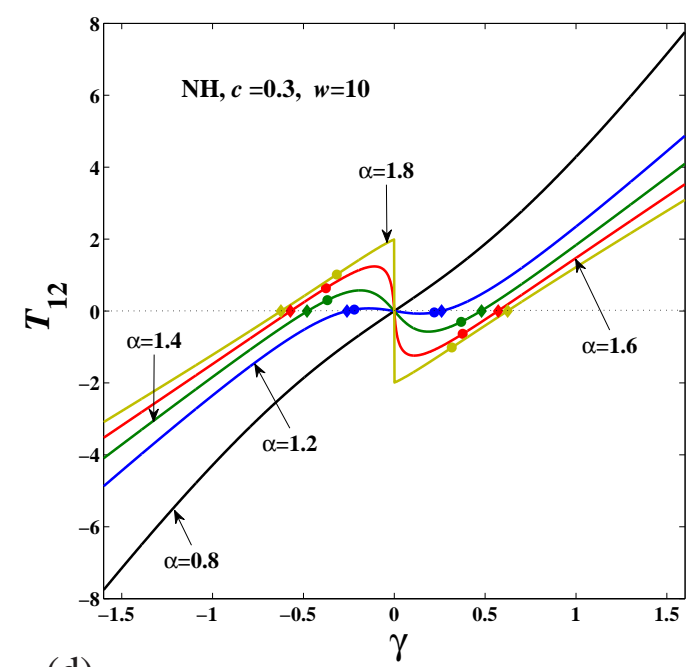

(d)

Figure 4: Principal solution for the behavior of fiber-reinforced, neo-Hookean elastomers with $c=0.3$ and $w=10$, as functions of $\gamma$ for prescribed values of $\alpha$. (a) Effective energy $\widehat{\Psi}$, (b) total rotation of the fibers $\phi=\varphi+\psi$, (c) normal stress difference $T_{11}-T_{22}$, (d) shear stress $T_{12}$. 
of the applied shear. For the more extreme cases corresponding to $\alpha=1.8$ and $\alpha=2$, the fibers undergo an abrupt (finite) rotation (i.e., they undergo a collective "flopping" motion) by application of only an infinitesimal amount of shear $\gamma$ away from $\gamma=0$, which translates into the release of a finite amount of energy, which is manifested as a "downward" kink in the energy function at $\gamma=0$. (The small vertical segments emanating from the kink in the energies in Figure 4(a) correspond to the values of energy associated with the multiple values of the particle rotation in the discontinuous region of Fig. 4(b), with the highest value of the energy corresponding to a zero value of the particle rotation-and are only shown for emphasis.) Next, Figure 4(c) and (d) provide plots for the associated Cauchy stress measures $T_{11}-T_{22}$ and $T_{12}$, as defined by expressions (47) and (48), respectively. The plots for the stress measure $T_{11}-T_{22}$ have very similar behaviors to those of the corresponding energy plots in Figure 4(a), with the difference that two (symmetric) minima of $T_{11}-T_{22}$ can fall outside or inside the non-convex region, depending on the value of $\alpha$. Finally, we observe from Fig. 4(d) that the Cauchy shear stress $T_{12}$ first increases and then decreases with increasing $\gamma$ inside the non-convex region, leading to the development of negative shear stresses with the application of positive shear strains, and vice versa, inside the non-convex region. In such regions, the material develops negative stiffness and naturally one expects the possible development of instabilities, including loss of ellipticity.

For completeness, Figure 5 shows plots of the critical stretches at which the energy function $\widehat{\Psi}$ first loses global convexity (with respect to $\alpha$ and $\gamma$ ) and strong ellipticity, respectively, under general loading conditions. Thus, Figure 5(a) shows plots of the critical curves for loss of global convexity and strong ellipticity, as functions of $\alpha$ and $\gamma$, for $c=0.3$ and $w=3.5,5$ and 10. (The circle and diamond markers correspond to the loss of strong ellipticity and global convexity, respectively.) An interesting observation from this figure is that the critical surfaces for the loss of SE close up as $\gamma$ increases, implying that the principal solution is (locally) strongly elliptic outside of this closed region (although not on the line $\gamma=0$, where the energy has a kink with negative curvature). On the other hand, the critical surfaces for the loss of global convexity do not close up, and instead exhibit an asymptotic behavior as $\gamma$ increases. Figure 5(b) provides a plot of the critical stretch $\alpha_{c r}$ (at which the energy first loses strong ellipticity for $\gamma=0$ ), as a function of the fiber volume fraction $c$ and aspect ratio $w$. This figure demonstrates that the energy function $\widehat{W}$ for the composite loses ellipticity sooner (at lower stretches) with increasing values of $c$ and $w$, in agreement with the results presented in Lopez-Pamies and Ponte Castañeda (2006b). In particular, composites with circular fibers $(w=1)$ never lose ellipticity (i.e., $\alpha_{c r} \rightarrow \infty$ as $w \rightarrow 1$ ). 

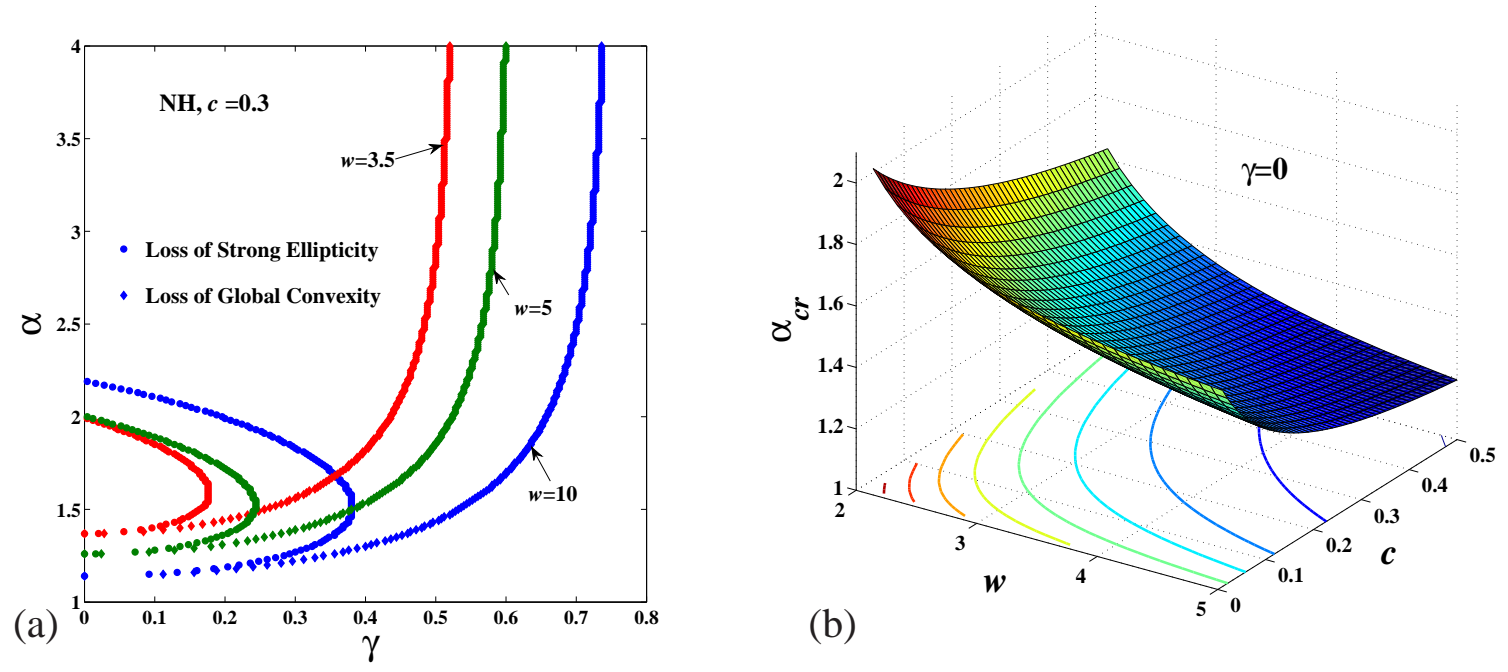

Figure 5: Estimates for the loss of (local) strong ellipticity (SE) and loss of global convexity (GC) in fiber-reinforced, neo-Hookean elastomers subjected to plane deformations. Part (a) shows results for the critical surfaces in the space $(\alpha, \gamma)$ at which loss of SE and GC of the principal energy function $\widehat{\Psi}(\alpha, \gamma)$ takes place. Part(b) shows results for the critical stretch $\alpha_{c r}$ (at which loss of SE of the principal energy function first takes place on the line $\gamma=0$ ), as a function of $c$ and $w$.

\section{Post-bifurcation solution and quasiconvexification of the energy}

In the previous section, we discussed the "principal" or "mesoscopic" response of the fiber-reinforced elastomer depicted in Fig. 2. Thus, it was seen that the stored-energy function $\widehat{W}$, determined by expression (38), develops "macroscopic" instabilities, corresponding to loss of strong ellipticity, when sufficiently large amounts of compressive deformation are applied along the long axis of the fibers. This suggest that the stored-energy function $\widehat{W}$ may also lose (global) rankone convexity - and therefore quasiconvexity - for such deformations. As a consequence, beyond these instabilities, the local deformation fields associated with the "principal" solution for the macroscopic response $\widehat{W}$ of the fiber-reinforced elastomer are expected to develop "microstructures" (minimizing sequences), by breaking up into "domains" tending to lower the energy in the composite. In other words, the effective stored-energy function for the composite $\widetilde{W}$, as defined by (13), is expected to be identical to $\widehat{W}$, up to the possible development of an instability, beyond which the principal solution bifurcates into some other post- 
bifurcation solution, such that $\widetilde{W} \leq \widehat{W}$. In this section, we are concerned with the characterization of the post-bifurcation, or "relaxed" stored-energy function $\widetilde{W}$, which is known to be quasiconvex, and therefore, rank-one convex. As discussed in Subsection 2.3, $\widetilde{W}$ will be estimated directly from the quasiconvexification of the "principal" solution $\widehat{W}$, as given by expression (20).

\subsection{Rank-1 convexification and relaxation of the energy}

In general, the direct computation of the quasiconvexification $Q \widehat{W}$ of the energy by means of expression (15) is not feasible. For this reason, it is of interest to also consider the so-called rank-one convex envelope of the energy (Dacorogna, 2007), as determined by

$$
R \widehat{W}(\mathbf{F})=\sup \{W(\mathbf{F}): W \leq \widehat{W} \text { and } W \text { rank-one convex }\},
$$

and with the property that

$$
Q \widehat{W}(\mathbf{F}) \leq R \widehat{W}(\mathbf{F}) .
$$

(It is recalled in this context that Conti (2008) has shown that quasiconvexity implies rank-one convexity for incompressible elastic materials.) In fact, it is sometimes the case that $R \widehat{W}$ can be shown to be polyconvex, and therefore, if this is the case, it follows by the result (12) that

$$
Q \widehat{W}(\mathbf{F})=R \widehat{W}(\mathbf{F}) .
$$

Furthermore, the computation of $R \widehat{W}$ is sometimes feasible, in particular, thanks to the iterative formula of Kohn and Strang (1986), which essentially amounts to a sequential lamination procedure involving $\widehat{W}$. More specifically, the result is

$$
R \widehat{W}(\mathbf{F})=\lim _{k \rightarrow \infty} R_{k} \widehat{W}(\mathbf{F}),
$$

where $R_{0} \widehat{W}(\mathbf{F})=\widehat{W}(\mathbf{F})$, and $R_{k} \widehat{W}(\mathbf{F})$ is defined recursively via

$$
R_{k} \widehat{W}(\mathbf{F})=\inf _{c^{(\mathrm{II})}, \mathbf{w}, \mathbf{N}}\left\{c^{(\mathrm{I})} R_{k-1} \widehat{W}\left(\mathbf{F}-c^{(\mathrm{II})} \mathbf{w} \otimes \mathbf{N}\right)+c^{(\mathrm{II})} R_{k-1} \widehat{W}\left(\mathbf{F}+c^{(\mathrm{I})} \mathbf{w} \otimes \mathbf{N}\right)\right\}
$$

Here $c^{(\mathrm{I})}=1-c^{(\mathrm{II})}, 0 \leq c^{(\mathrm{II})} \leq 1$, and $\mathbf{N}$ and $\mathbf{w}$ are arbitrary vectors (in the plane of the deformation). Thus, it can be seen that the rank-one convexification process makes use of a special subset of the minimizers in the definition (15) of the quasiconvexification $Q \widehat{W}$, namely those leading to rank-one connected deformation 
gradients in the layers, which play the role of the "domains" in the more general result.

Next, we consider in more detail the first iteration $(k=1)$ in the above procedure, which is given by

$$
R_{1} \widehat{W}(\mathbf{F})=\inf _{c^{(\mathrm{II})}, \mathbf{w}, \mathbf{N}}\left\{c^{(\mathrm{I})} \widehat{W}\left(\mathbf{F}-c^{(\mathrm{II})} \mathbf{w} \otimes \mathbf{N}\right)+c^{(\mathrm{II})} \widehat{W}\left(\mathbf{F}+c^{(\mathrm{I})} \mathbf{w} \otimes \mathbf{N}\right)\right\}
$$

and note that it can be rewritten in the form

$$
R_{1} \widehat{W}(\mathbf{F})=\inf _{c^{(\mathrm{II})}, \mathbf{N}} \widehat{W}_{L}\left(\mathbf{F} ; c^{(\mathrm{II})}, \mathbf{N}\right),
$$

where

$$
\widehat{W}_{L}\left(\mathbf{F} ; c^{(\mathrm{II})}, \mathbf{N}\right)=\inf _{\boldsymbol{\omega}}\left\{c^{(\mathrm{I})} \widehat{W}\left(\mathbf{F}\left(\mathbf{I}-c^{(\mathrm{II})} \boldsymbol{\omega} \otimes \mathbf{N}\right)\right)+c^{(\mathrm{II})} \widehat{W}\left(\left(\mathbf{F}\left(\mathbf{I}+c^{(\mathrm{I})} \boldsymbol{\omega} \otimes \mathbf{N}\right)\right)\right\}\right.
$$

is the homogenized stored-energy function of a laminated composite made up of two (identical) anisotropic phases with energy function $\widehat{W}$, in concentrations $c^{(\mathrm{I})}=1-c^{(\mathrm{II})}$ and $c^{(\mathrm{II})}$, and layered in the direction specified by the unit vector $\mathbf{N}$. (Note that we have made the change of variables $\boldsymbol{\omega}=\mathbf{F}^{-1} \mathbf{w}$.) In connection with expression (57), it should be noted that $\boldsymbol{\omega}=\mathbf{0}$, corresponding to a uniform deformation $\mathbf{F}$ in the laminate, is always a possible solution; however, lower energy solutions may be possible in some cases by piecewise uniform deformations satisfying the compatibility and equilibrium requirement across the layer interfaces, as well as the average condition implicit in this result (deBotton, 2005; LopezPamies and Ponte Castañeda, 2009). Thus, it is seen from equation (56) that $R_{1} \widehat{W}$ corresponds to the lowest energy that may be obtained by layering in all possible directions and in all possible volume fractions $c^{(\mathrm{II})}$.

For the fiber-reinforced elastomers of interest here, the energy function $\widehat{W}$ is defined by (38) in terms of $\widehat{\Phi}$, where it is recalled that $\operatorname{det} \mathbf{F}=1$, due to the incompressibility of the phases. It follows from this last fact that

$$
\operatorname{det}\left(\mathbf{F}\left(\mathbf{I}-c^{(\mathrm{II})} \boldsymbol{\omega} \otimes \mathbf{N}\right)\right)=\operatorname{det}\left(\mathbf{I}-c^{(\mathrm{II})} \boldsymbol{\omega} \otimes \mathbf{N}\right)=1-c^{(\mathrm{II})} \boldsymbol{\omega} \cdot \mathbf{N}=1,
$$

(and similarly for the other term), and therefore that $\boldsymbol{\omega} \cdot \mathbf{N}=0$. Letting $\mathbf{M}$ be a unit vector orthogonal to the unit vector $\mathbf{N}$ in the plane of the deformation, we write that

$$
\boldsymbol{\omega}=\varpi \mathbf{M}
$$




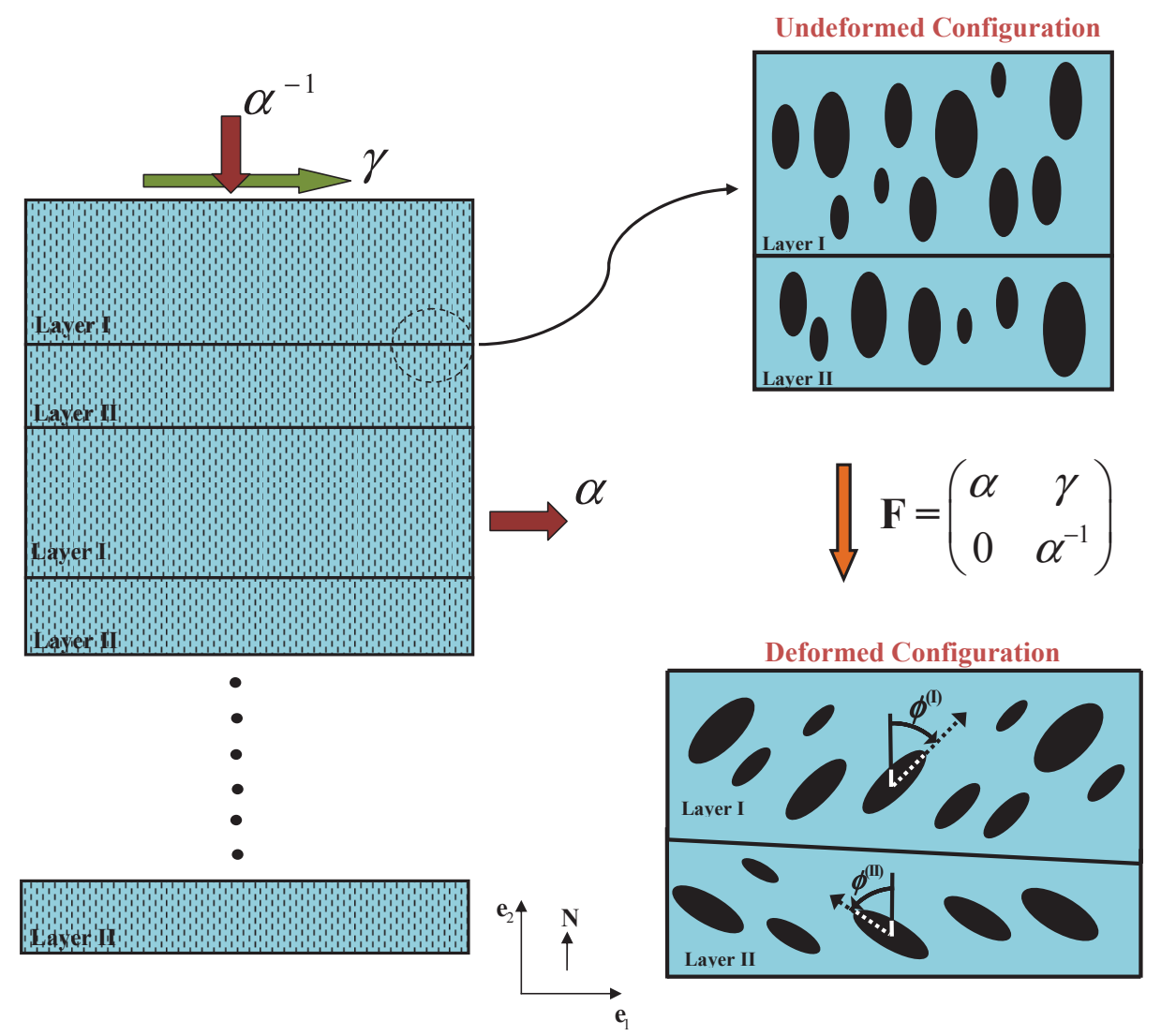

Figure 6: Schematic illustration of the rank-one lamination

where $\varpi$ is the magnitude of $\boldsymbol{\omega}$. In this case, we can rewrite the result (55) for the first iteration in the rank-one convexification as

$R_{1} \widehat{W}(\mathbf{F})=\min _{\mathbf{N}, \varpi, c^{(\mathrm{II})}}\left[c^{(\mathrm{I})} \widehat{W}\left(\mathbf{F}\left(\mathbf{I}-c^{(\mathrm{II})} \varpi \mathbf{M} \otimes \mathbf{N}\right)\right)+c^{(\mathrm{II})} \widehat{W}\left(\mathbf{F}\left(\mathbf{I}+c^{(\mathrm{I})} \varpi \mathbf{M} \otimes \mathbf{N}\right)\right)\right]$.

A direct numerical calculation of expression (60) suggests that the rank-one convexification of $\widehat{W}$ is given by the convexification of the energy $\widehat{\Psi}$ in the subspace of deformation variables $\alpha$ and $\gamma$, namely,

$$
R_{1} \widehat{W}(\mathbf{F})=\widehat{\Psi}^{* *}(\alpha, \gamma)+K(\operatorname{det} \mathbf{F})
$$

where the superscript * refers to the Legendre-Fenchel transform and $K$ is defined by (39). To justify this result, we make the following observations. First, referring to the result (57) for the energy $\widehat{W}_{L}\left(\mathbf{F} ; c^{(\mathrm{II})}, \mathbf{N}\right)$ of a layered elastomer, it is recalled 
that the solution of this problem is the principal solution $\widehat{W}$ (for the homogeneous material) unless there is an instability leading to a bifurcation into a lower energy solution. Second, the principal solution $\widehat{W}$ is known to exhibit such an instability when the vector $\mathbf{N}$ in the loss of ellipticity condition (11) is aligned with the direction of the long fiber axis $\mathbf{e}_{2}$ (when $\gamma=0$ ). This suggests that non-trivial solutions of the laminated elastomer problem (57) may be expected to first take place in this case. Referring to Figure 6, the corresponding deformations in expression (60) can then be written in the form

$$
\mathbf{F}^{(\mathrm{I})}=\mathbf{F}\left(\mathbf{I}-c^{(\mathrm{II})} \varpi \mathbf{e}_{1} \otimes \mathbf{e}_{2}\right), \quad \text { and } \quad \mathbf{F}^{(\mathrm{II})}=\mathbf{F}\left(\mathbf{I}+c^{(\mathrm{I})} \varpi \mathbf{e}_{1} \otimes \mathbf{e}_{2}\right),
$$

where we have used the fact that $\mathbf{M}=\mathbf{e}_{1}$. In matrix notation, relative to the laboratory coordinates,

$$
F_{i j}^{(\mathrm{I})}=\left(\begin{array}{cc}
\alpha & \gamma-c^{(\mathrm{II})} \alpha \varpi \\
0 & \alpha^{-1}
\end{array}\right), \quad \text { and } \quad F_{i j}^{(\mathrm{II})}=\left(\begin{array}{cc}
\alpha & \gamma+c^{(\mathrm{I})} \alpha \varpi \\
0 & \alpha^{-1}
\end{array}\right) .
$$

It can then be shown that minimizing expression (60) for $R_{1} \widehat{W}$ over $\varpi$ and $c^{(\mathrm{II})}$ for $\mathbf{N}=\mathbf{e}_{2}$ corresponds to convexifying $\widehat{\Psi}$ with respect to $\gamma$ (for fixed $\alpha$ ), leading to the result (61). In this connection, it should be emphasized that while the normal to the shear band in the loss of strong ellipticity condition depends on the amount of shear $\gamma$, the normal to the layers in the rank-one convexification calculation is independent of $\gamma\left(\mathbf{N}=\mathbf{e}_{2}\right)$. Thus the two results agree only for $\gamma=0$, which will be seen later to be a consequence of the fact that the reinforced elastomers generally lose global rank-one convexity before they lose local rank-one convexity, except at $\gamma=0$ when both are lost simultaneously.

Referring to Fig. 7(a), it is recalled from Section 3.3 that $\widehat{\Psi}$ loses convexity for $-\gamma_{c r}^{C}<\gamma<\gamma_{c r}^{C}$, when $\alpha>\alpha_{c r}$, where $\alpha_{c r}$ is the value of $\alpha$ at which $\widehat{\Psi}$ loses convexity (and the material loses strong ellipticity according to condition (B.6)) for $\gamma=0$. Thus, when $\alpha>\alpha_{c r}$ is given and $\gamma$ is between the minima $\left(-\gamma_{c r}^{C}<\gamma<\right.$ $\left.\gamma_{c r}^{C}\right)$, the values of $\varpi$ and $c^{(\text {II })}$ should be chosen such that the shear strain in the two phases of the laminate (layers I and II) are precisely at the two minima (at $\gamma_{c r}^{C}$ and $\left.-\gamma_{c r}^{C}\right)$. Thus, as can be seen from Fig. $7(\mathrm{~b}), \varpi$ is constant and $c^{(\mathrm{II})}$ varies linearly between 0 and 1 , as $\gamma$ ranges between $-\gamma_{c r}^{C}$ and $\gamma_{c r}^{C}$. Furthermore, given $\alpha$ and the two shear strains in layers I and II, $\gamma_{c r}^{C}$ and $-\gamma_{c r}^{C}$, the corresponding values of $\lambda, \theta$ and $\psi$ in each layer can be computed from expressions (34) to (37), which in turn can be used to determine the value of the energy $\widehat{\Phi}$ and of the particle rotation $\varphi$ (in each layer) via (40) and (41), respectively. It is remarked that the "deformed" fiber rotation angles in layers I and II have the same magnitude, but opposite 


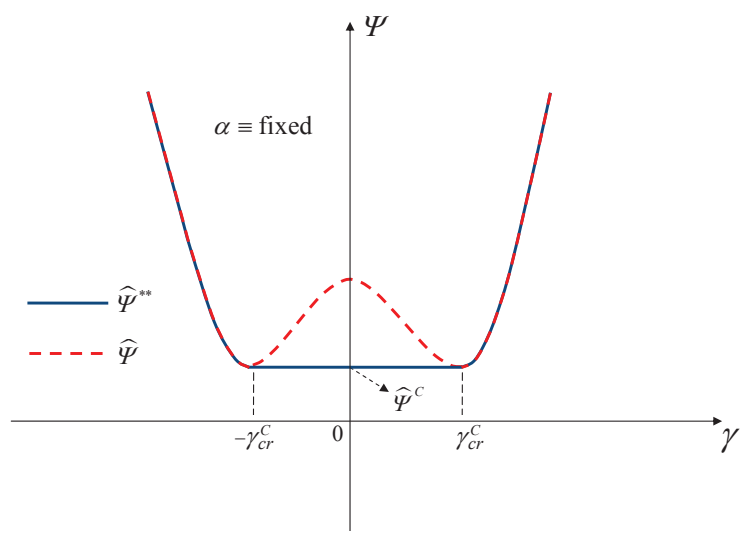

(a)

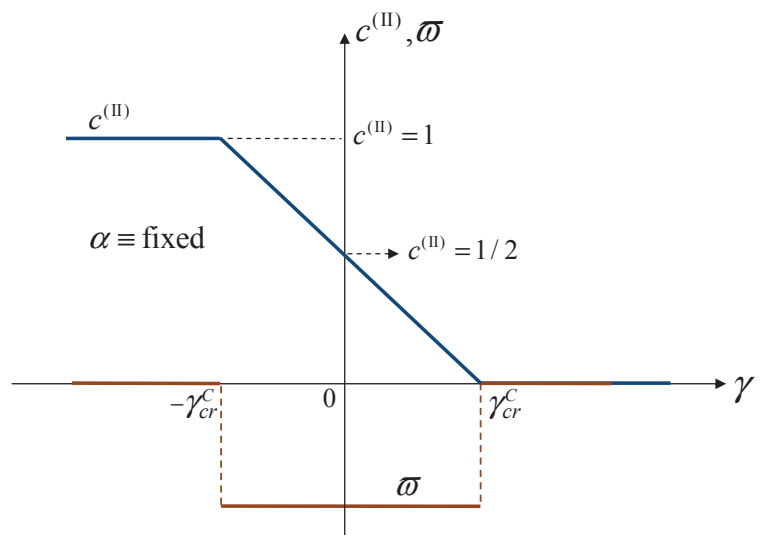

(b)

Figure 7: Schematic illustration of the solution to the minimization equation (60) under deformation (31) at a fixed $\alpha$. (a) Stored-energy functions for the principal solution and for the rank-one laminate (b) The optimized values for $c^{(\mathrm{II})}$ and $\varpi$.

signs, so that the fibers in the two layers are symmetrically oriented relative to the initial fiber direction (cf. Fig. 6). On the other hand, the stretch $\lambda$ in both layers is exactly the same, and the macroscopic amount of shear $\gamma$ is accommodated simply by varying the volume fractions of the two layers.

Of course, when $|\gamma|>\gamma_{c r}^{C}$, there is no layering (no development of microstructure), and the energy and particle rotation are the same as those of the principal solution. Note, however, that as $\gamma \rightarrow \pm \gamma_{c r}^{C}$ from the side of the non-convex region, the volume fraction of the layers, $c^{(\mathrm{I})}$ and $c^{(\mathrm{II})}$, tend to 0 and $100 \%$, so that the layered solution joins smoothly the principal solution at $|\gamma|=\gamma_{c r}^{C}$.

In any event, the stored-energy function $R_{1} \widehat{W}(\mathbf{F})$ of the rank-one laminate, as given by expression (61) in terms of the convexification of $\widehat{\Psi}$, can be determined from

$$
\widehat{\Psi}^{* *}(\alpha, \gamma)=\left\{\begin{array}{l}
\widehat{\Psi}^{C}(\alpha)=\widehat{\Psi}\left(\alpha, \pm \gamma_{c r}^{C}\right)=\min _{ \pm \gamma>0} \widehat{\Psi}(\alpha, \gamma), \quad \alpha>\alpha_{c r},|\gamma|<\gamma_{c r}^{C}, \\
\widehat{\Psi}(\alpha, \gamma), \quad \text { otherwise, }
\end{array}\right.
$$

where it is emphasized that $\widehat{\Psi}^{C}$ and $\gamma_{c r}^{C}$ are functions of the variable $\alpha$ only.

In summary, the rank-one lamination of the principal solution $R_{1} \widehat{W}$ is given by expression (61), together with (64). Now, as may be suspected from the convexity of $\widehat{\Psi}^{* *}$ on $\alpha$ and $\gamma$, it can be verified numerically that $R_{1} \widehat{W}$ is strongly elliptic (except at $\alpha=\alpha_{c r}$ and $\gamma=0$ where it just loses strong ellipticity), and is therefore 
rank-one convex. (It should also be emphasized that the deformation fields $\mathbf{F}^{(\mathrm{I})}$ and $\mathbf{F}^{(\mathrm{II})}$ in layers I and II are such that the response of the material in the layers is also strongly elliptic.) It follows from the result (53) that $R_{1} \widehat{W}$ must be the rank-one convex envelope of $\widehat{W}$, that is,

$$
R \widehat{W}(\mathbf{F})=R_{1} \widehat{W}(\mathbf{F})=\widehat{\Psi}^{* *}(\alpha, \gamma)+K(\operatorname{det} \mathbf{F}) .
$$

Moreover, the convexity of $\widehat{\Psi}^{* *}$ in the variables $\alpha$ and $\gamma$, together with the convexity of $K$ in $J=\operatorname{det} \mathbf{F}$, suggests that the function $R \widehat{W}(\mathbf{F})$ above may also be polyconvex. Indeed, it is shown in Appendix $\mathrm{C}$ that $R \widehat{W}=R_{1} \widehat{W}$, as given by expression (65), is in fact polyconvex. As already mentioned, it then follows from the hierarchy (12) that $R \widehat{W}=R_{1} \widehat{W}$ must also be quasiconvex, and therefore that

$$
\widetilde{W}(\mathbf{F})=Q \widehat{W}(\mathbf{F})=R \widehat{W}(\mathbf{F})=R_{1} \widehat{W}(\mathbf{F})
$$

is the quasiconvexification or relaxation of the energy corresponding to the storedenergy function of the fiber-reinforced elastomer in the post-bifurcation regime.

Having determined the relaxed stored-energy function $\widetilde{W}$ of the fiber-reinforced elastomers, we provide next expressions for the corresponding macroscopic stresses, generalizing expressions (47)-(48) into the post-bifurcation regime. Thus, the normal stress difference can be written in the form

$$
T_{11}-T_{22}=\left\{\begin{array}{l}
T_{11}^{C}-T_{22}^{C}, \quad-\gamma_{c r}^{C}<\gamma<\gamma_{c r}^{C}, \alpha>\alpha_{c r}, \\
\text { equation (47), otherwise, }
\end{array}\right.
$$

where $T_{11}^{C}-T_{22}^{C}$ depends only on $\alpha$ (i.e., it is constant for $-\gamma_{c r}^{C}<\gamma<\gamma_{c r}^{C}$ ), and it can be computed most easily from expression (47) by setting

$$
T_{11}^{C}-T_{22}^{C}=\left(T_{11}-T_{22}\right)\left(\alpha, \gamma_{c r}^{C}(\alpha)\right) \text {. }
$$

On the other hand, the corresponding Cauchy shear stress is given by

$$
T_{12}=\left\{\begin{array}{l}
0, \quad-\gamma_{c r}^{C}<\gamma<\gamma_{c r}^{C}, \alpha>\alpha_{c r}, \\
\text { equation (48), otherwise. }
\end{array}\right.
$$

It is emphasized that, as a consequence of this last result, the response of the reinforced elastomer (in the relaxed regime) is perfectly soft (i.e., it has a zero shear modulus) in shear transverse to the long fiber direction. On the other hand, as we will see in the next section showing some numerical examples, the response to pure shear deformations with compression along the long fiber direction is softer in the post-bifurcation regime than the principal solution, but has a finite shear modulus. 


\subsection{Representative examples of the post-bifurcation estimates}

In this subsection, we present some representative results of the "relaxed" or post-bifurcation estimates for the macroscopic response of, and microstructure evolution in, fiber-reinforced elastomers consisting of an incompressible matrix phase and rigid fibers (with elliptical cross-section) undergoing plane deformations of the form (31). For consistency of notation, we introduce the relaxed energy function $\widetilde{\Psi}=\widehat{\Psi}^{* *}$, such that $\widetilde{W}(\mathbf{F})=\widetilde{\Psi}(\alpha, \gamma)+K(J)$. Most of the results given in this section are for neo-Hookean elastomers, but some results are also given for Gent elastomers at the end of this section to explore the effect of strain locking on the onset of the instability and post-bifurcation results. We will first present results to elucidate the main features of the estimates, and then explore the effects of the fiber volume fraction $c$ and aspect ratio $w$. The results for the energies, as well as for the stresses, will be normalized by the ground-state shear modulus of the elastomer (i.e., $\mu^{(1)}=1$ ). Finally, we recall that the circle ' $\bullet$ and diamond ' $\bullet$ markers are used in the plots to label the points where the principal solution loses (local) strong ellipticity and (global) rank-one convexity, respectively.

Figure 8 shows a 3-D landscape of the relaxed stored-energy energy function $\widetilde{\Psi}$ of the fiber-reinforced neo-Hookean elastomer in the deformation variables $\alpha$ and $\gamma$, calculated for fixed values $c=0.3$ and $w=10$. This figure is the counterpart of Figure 3, which shows the corresponding landscape for the principal solution for the energy function $\widehat{\Psi}$. As is evident from comparing these two plots, the crease region in the principal energy landscape (that was identified earlier by the interval $\left[-\gamma_{c r}^{C}, \gamma_{c r}^{C}\right]$, where $\widehat{\Psi}$ loses global convexity in the variables $\alpha$ and $\gamma$ ) is replaced by the convex hull for the relaxed energy function $\widetilde{\Psi}$.

Figure 9 presents more detailed results for the relaxed energy and associated microstructural variables for a fiber-reinforced neo-Hookean elastomer with $c=0.3$ and $w=10$. Corresponding results for the principal solution (PS) are also shown for comparison. All the results in this figure are presented as functions of $\gamma$ for several, fixed values of $\alpha$. Figure 9(a) shows that the relaxed or post-bifurcation solution (PBS) is the convex hull of the principal solution with the relaxed values lying well below the principal values in the interval $\left[-\gamma_{c r}^{C}, \gamma_{c r}^{C}\right]$ (denoted by diamond markers). As depicted in Figure 9(b-d), the relaxation of the energy takes place by the formation of laminates with two types of layers consisting of the same material, but with equal and opposite fiber orientations $\pm \phi$ (that are fixed for given $\alpha$ in the relaxed region), with concentrations $c^{(\text {II) }}$ and $c^{(\mathrm{I})}=1-c^{(\mathrm{II})}$, that vary linearly from $0 \%$ to $100 \%$ and vice versa, as $\gamma$ decreases 


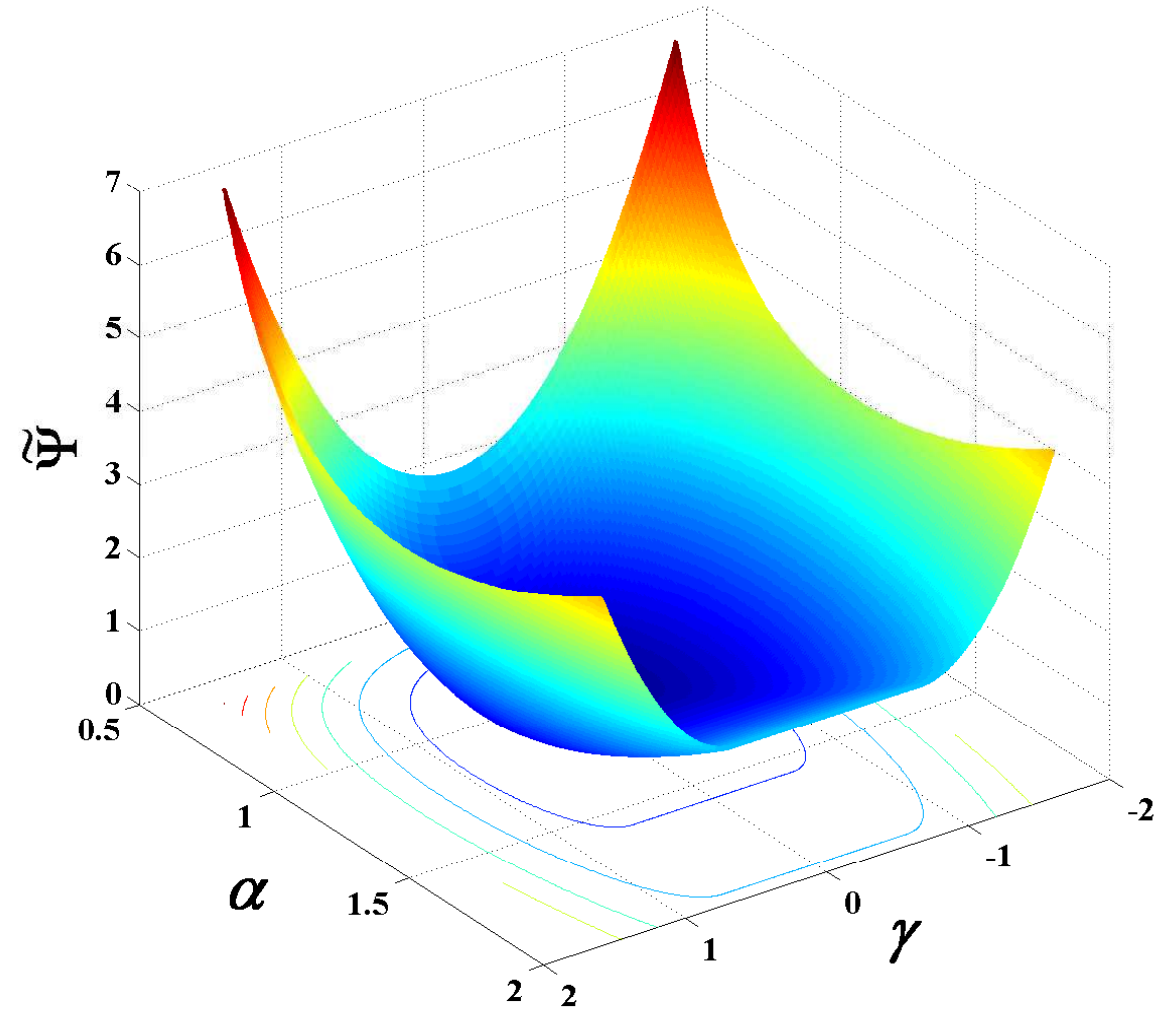

Figure 8: Estimate for the relaxed stored-energy function $\widehat{\Psi}$ of fiber-reinforced, neo-Hookean elastomers with $c=0.3$ and $w=10$, as functions of loading variables $\alpha$ and $\gamma$.

(from right to left). The relaxed solution joins smoothly the principal solution outside the non-convex region by matching the fiber orientation (in the layers that reach $100 \%$ volume fraction) to that of the principal solution just outside. Thus, we observe from Figure 9(c) that the volume fraction of the layers of type II are zero for sufficiently large values of $\gamma>0$, implying that the relaxed solution reduces to the principal solution for large enough values of $\gamma\left(\gamma>\gamma_{c r}^{C}\right)$. However, once $\gamma$ decreases to the critical value $\gamma_{c r}^{C}$, layers of type II are "nucleated" and their volume fraction increases linearly as $\gamma$ decreases further until the value $\gamma=-\gamma_{c r}^{C}$ is reached, where $c^{(\mathrm{II})}$ in turn reaches $100 \%$. Beyond this point (i.e., when $\gamma<-\gamma_{c r}^{C}$ ), the volume fraction of the layers of type I is zero, and the relaxed solution again reduces to the principal solution. It is also interesting to note that the size of the 


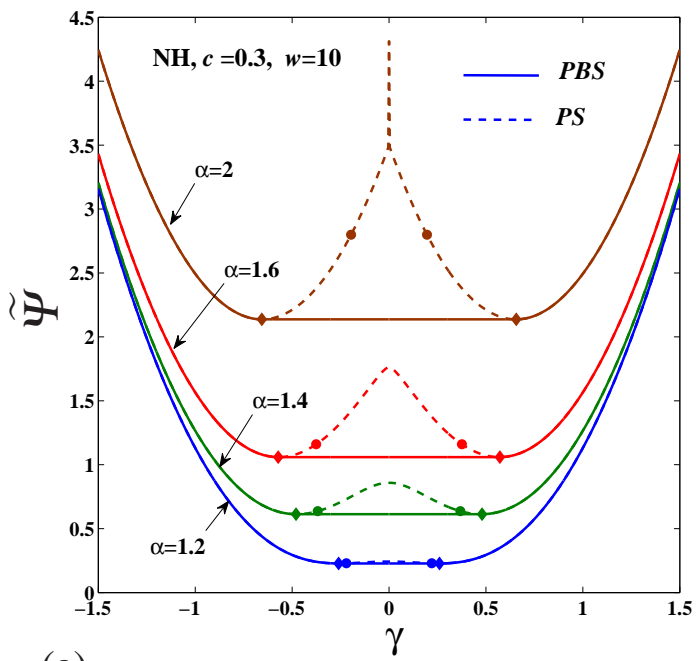

(a)

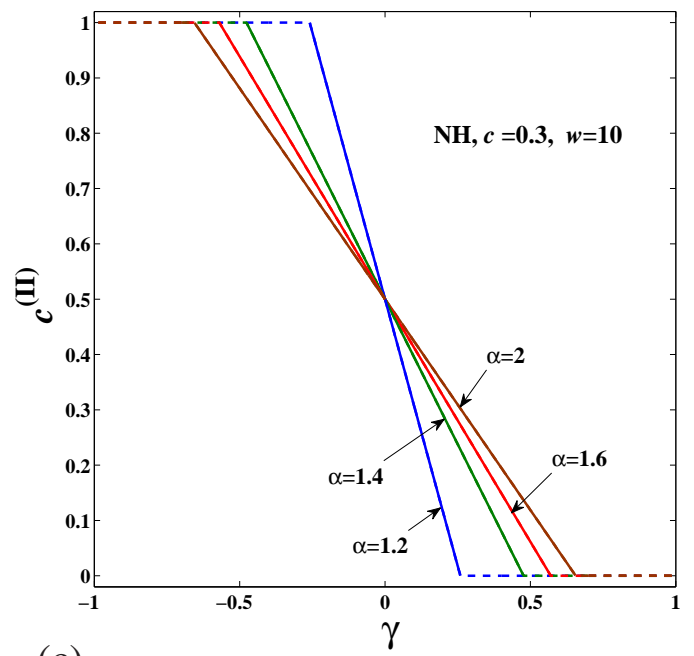

(c)

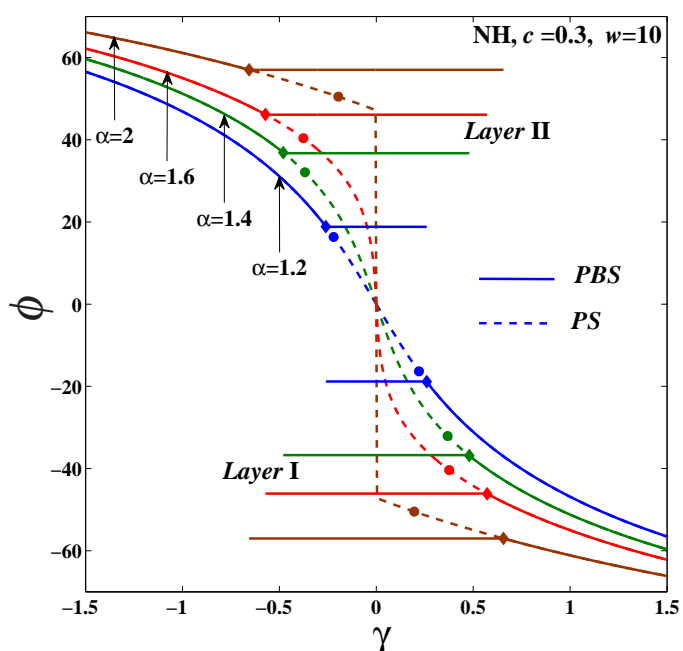

(b)

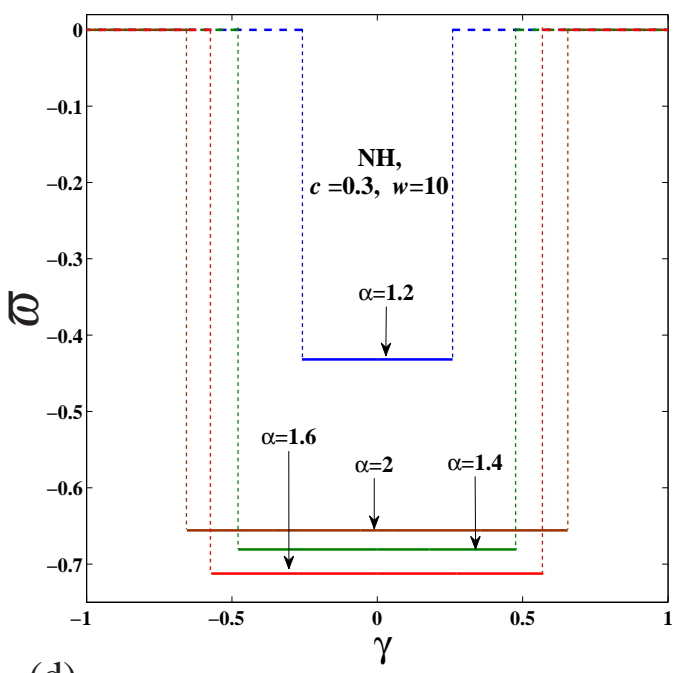

(d)

Figure 9: Relaxed estimates for the macroscopic and microscopic response of fiber-reinforced, neo-Hookean elastomers with $c=0.3$ and $w=10$, as functions of $\gamma$, for prescribed values of $\alpha$. (a) Effective energy $\widetilde{\Psi}$, (b) (total) rotation of the fibers in layers I and II. (The principal estimates are also included for comparison.) (c) and (d) The corresponding values for layer volume fraction $c^{(\mathrm{II})}$ and amplitude vector $\varpi$ associated with the relaxed solution. 


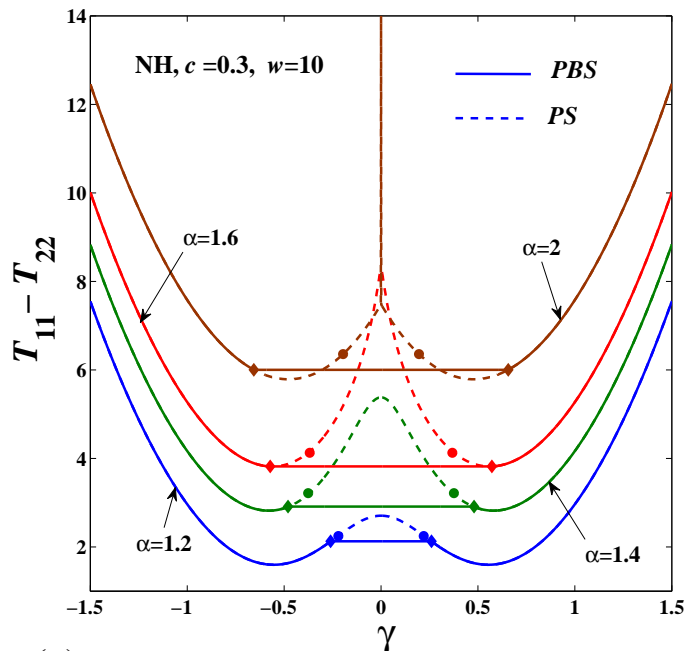

(a)

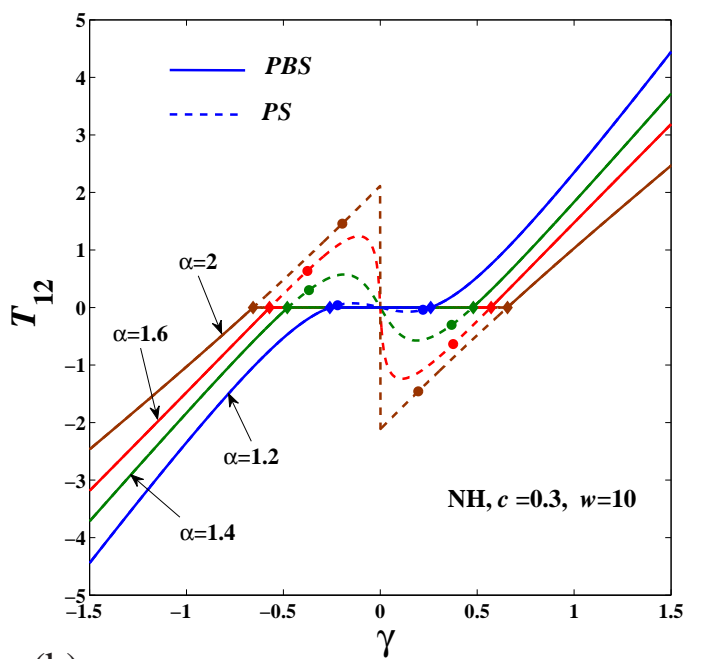

(b)

Figure 10: Relaxed estimates for the macroscopic stresses of fiber-reinforced neo-Hookean elastomers with $c=0.3$ and $w=10$, as functions of $\gamma$, for prescribed values of $\alpha$. (a) normal Cauchy stress difference $T_{11}-T_{22}$, (b) Cauchy shear stress $T_{12}$. The corresponding principal estimates are also included for comparison.

relaxed region increases monotonically with increasing values of $\alpha$, which also leads to an increase in the magnitudes of the fiber rotations, but slower changes in the layer concentrations with increasing $\gamma$. On the other hand, the magnitude of the amplitude vector $\Phi$ is not monotonic in $\alpha$, but it should be kept in mind from equations (63) that the total jump in the deformation gradient also depends (linearly) on $\alpha$.

Figure 10 presents results for the relaxed estimates (PBS) for the stresses in fiber-reinforced neo-Hookean elastomers with $c=0.3$ and $w=10$, as a function of $\gamma$, for several values of $\alpha$. The principal estimates (PS) are also shown for comparison. Figures 10(a) and (b) provide estimates for the normal Cauchy stress difference $T_{11}-T_{22}$ and Cauchy shear stress $T_{12}$, respectively. We observe from these figures that the relaxed stresses are generally softer than the corresponding principal stresses in the interval $\left[-\gamma_{c r}^{C}, \gamma_{c r}^{C}\right]$. In particular, the stress measure $T_{11}-T_{22}$ takes a non-zero constant value, which is typically well below the corresponding principal solution in this interval, while the Cauchy shear stress $T_{12}$ remains zero for the entire interval. These results are consistent with the general expectation that the instabilities lead to softer modes of deformation by the for- 


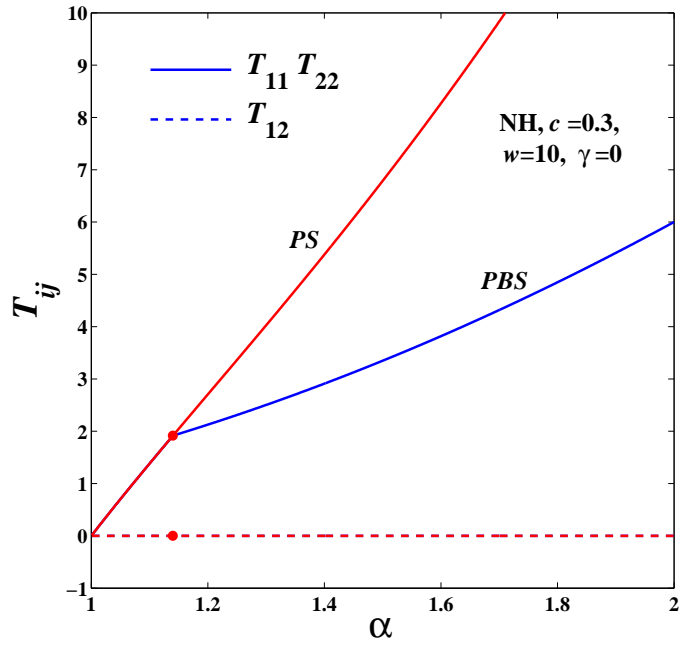

(a)

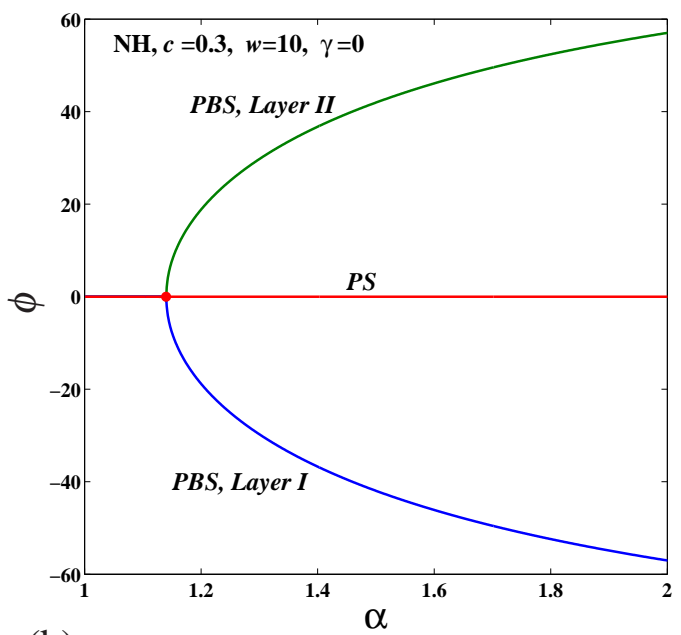

(b)

Figure 11: Relaxed estimates for the macroscopic stresses and rotation of the fibers in fiberreinforced neo-Hookean elastomers with $c=0.3$ and $w=10$. Results are shown for "aligned" loadings $(\gamma=0$ ), as functions of $\alpha$. (a) Stresses (b) Fiber rotation. The corresponding principal estimates are also included for comparison.

mation of (layered) microstructures. Indeed, it can be seen that the corresponding moduli in the relaxed regions vanish identically (in this mode of deformation).

Next, Figure 11 shows plots for the relaxed and principal estimates for the macroscopic stresses and associated fiber rotations in fiber-reinforced neo-Hookean elastomers for fixed values $c=0.3$ and $w=10$. The results are for "aligned" loadings (i.e, $\gamma=0$ ), and are presented as functions of $\alpha$. As can be seen from Figure 11(a), the principal solution (PS) for the normal stress difference bifurcates into the post-bifurcation solution (PBS), which is significantly less stiff than the principal solution. Correspondingly, Figure 11(b) shows that the principal solution, where the all the fibers remain perfectly aligned (they have zero rotation), bifurcates into the relaxed solution by the sudden formation of laminates with two types of layers in equal volume fractions, where the fibers assume finite reorientation angles that are equal in magnitude and opposite in sign. The layers in the post-bifurcated or relaxed solution are "nucleated" precisely at the point where the strong ellipticity (labelled by the circular marker) of the principal solution is lost, and thus the fibers start rotating to accommodate the macroscopic deformation when the shear modulus transverse to the long axis of the fibers vanishes. 


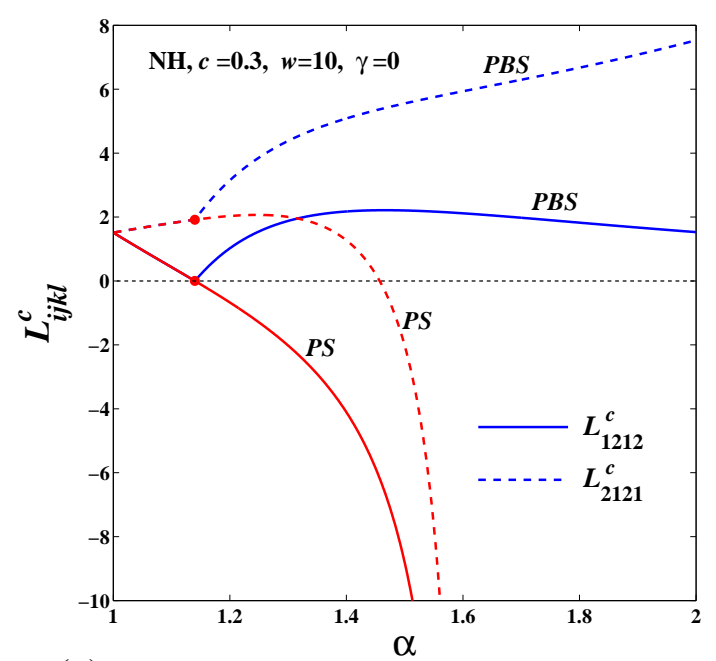

(a)

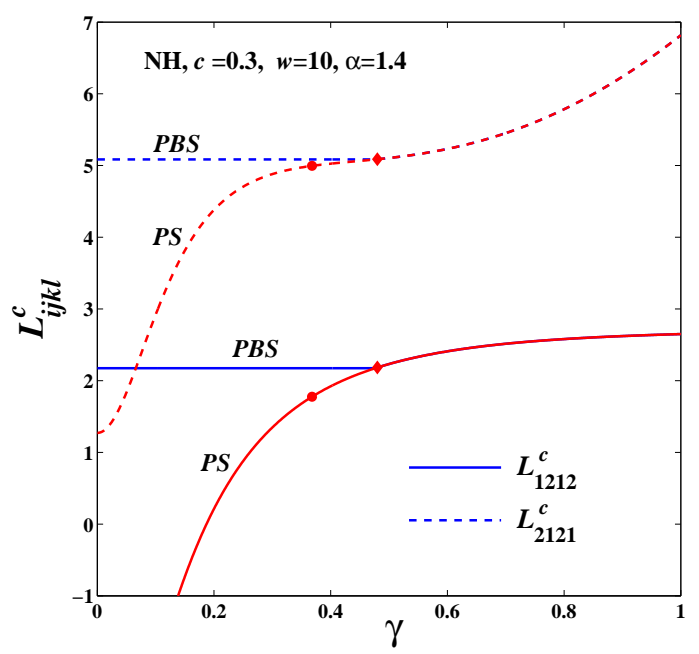

(c)

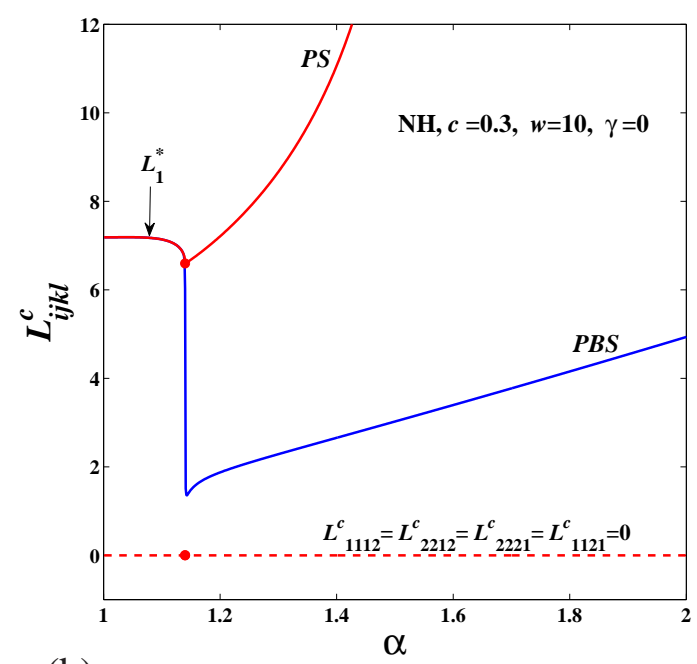

(b)

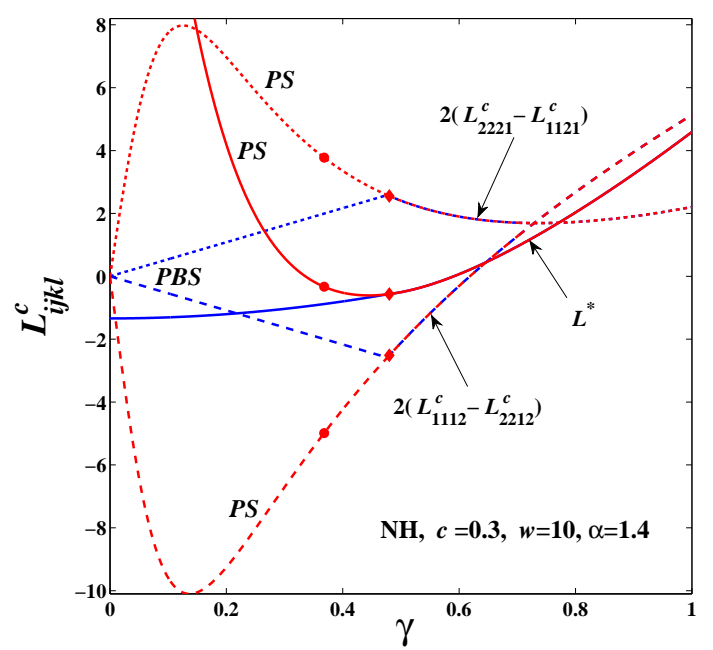

(d)

Figure 12: Relaxed estimates for the relevant traces of the tangent modulus tensor $\mathbf{L}^{c}$ in fiberreinforced, neo-Hookean elastomers with $c=0.3$ and $w=10$. Parts (a-b) shows results for aligned loadings $(\gamma=0)$, as functions of $\alpha$. Parts (c-d) shows results for the fixed value $\alpha=1.4$, as functions of $\gamma$. The corresponding principal estimates are also included for comparison. 
Figure 12 presents plots for the five traces of the (instantaneous) elasticity tensor $\mathbf{L}^{c}$ appearing in the strong ellipticity condition (B.3). Figure 12(a) and (b) show results for the case of aligned loadings (i.e., $\gamma=0$ ), as a function of $\alpha$, while Figure 12(c) and (d) show the corresponding results, as a function of $\gamma$, at the fixed stretch $\alpha=1.4$. In particular, Figures 12 (a) and (c) show plots for moduli components $L_{1212}^{c}$ and $L_{2121}^{c}$, while Figures 12 (b) and (d) show plots for the traces $L^{*}=L_{1111}^{c}+L_{2222}^{c}-2\left(L_{1122}^{c}+L_{1221}^{c}\right), 2\left(L_{1112}^{c}-L_{2212}^{c}\right)$, and $2\left(L_{2221}^{c}-L_{1121}^{c}\right)$. (In accord with expression (B.5) for aligned loadings, Figure 12(b) shows results for $L_{1}^{*}=0.5 L^{*}+\sqrt{L_{1212}^{c} L_{2121}^{c}}$ instead of $L^{*}$.) Consistent with the discussion in Appendix $\mathrm{B}$ for loss of strong ellipticity of the principal solution under aligned loading conditions, we observe from Figure 12(a) and (b) that, as $\alpha$ increases, the shear modulus $L_{1212}^{c}$ (as predicted by the principal solution) reaches zero before $L_{2121}^{c}$, leading to development of a shear band whose normal is aligned with $\mathbf{e}_{2}$ and has slip direction $\mathbf{e}_{1}$. In contrast, the moduli $L_{1212}^{c}, L_{2121}^{c}$ and $L_{1}^{*}$, associated with the relaxed solution, never vanish (except at the onset of the bifurcation, when $\alpha=\alpha_{c r}$ ), and therefore the relaxed solution remains strongly elliptic for the entire range of $\alpha\left(\alpha>\alpha_{c r}\right)$. In particular, the moduli trace $L_{1}^{*}$ exhibits a discontinuity at $\alpha=\alpha_{c r}$ which is related to the fact that the curvature of the relaxed energy function (for $\gamma=0$ ) changes at the point of bifurcation from the corresponding principal energy function. Next, Figure 12(c) and (d) show the variation of the corresponding moduli traces as functions of $\gamma$. We recall that when $\gamma \neq 0$ the strong ellipticity condition is more involved because the off-diagonal traces $2\left(L_{1112}^{c}-L_{2212}^{c}\right)$ and $2\left(L_{2221}^{c}-L_{1121}^{c}\right)$ are generally non-zero. In this case, the loss of strong ellipticity takes place if the quartic polynomial in (B.3) admits at least one real root. Therefore, it is necessary to investigate the four possible roots of the quartic polynomial in (B.3) in order to examine the strong ellipticity of the relaxed and principal solutions for the case of $\gamma \neq 0$. For brevity, the results are not shown, but it has been verified that the imaginary parts associated with the relaxed solution remain non-zero for the entire range of deformations, implying that the relaxed solution is strongly elliptic. (We have also verified that the solution remains strongly elliptic in the layers.)

Next, we investigate the influence of the fiber volume fraction, aspect ratio, and constitutive properties of the matrix on the estimates for the relaxed response of the fiber-reinforced elastomers. In some of the results presented in the figures, use is made of the nominal stress measure $S$, which is defined in terms of the normal components of the Cauchy stress tensor by

$$
S=\frac{1}{\alpha}\left(T_{11}-T_{22}\right)
$$


Figure 13 provides results for the relaxed estimates for the response of the fiber-reinforced neo-Hookean elastomers for several fiber volume fractions ( $c=$ $0.01,0.05,0.15,0.3)$, at a fixed aspect ratio $(w=10)$. Figures 13(a) and (b) shows plots for the normal stress difference $S$ and the particle rotation $\phi^{(\mathrm{I})}$, respectively, as functions of $\alpha$, for aligned loadings $(\gamma=0)$. We observe from Figure 13(a) that increasing the fiber volume fraction $c$ leads to more pronounced softening (i.e., sharper drops in the incremental modulus) at the critical value of the stretch (i.e., when $\alpha=\alpha_{c r}$ ), although the response of the composite remains stiffer overall for higher values of $c$ beyond the bifurcation instability. Making contact with the evolution of the microstructure, we can see from Figure 13(b) that the abrupt drop in stiffness is induced by the sudden rotation of fibers at the associated value of $\alpha=\alpha_{c r}$. (Note that in this case $c^{(\mathrm{I})}=c^{(\mathrm{II})}=0.5$ and $\phi^{(\mathrm{II})}=-\phi^{(\mathrm{I})}$ for entire range of $\alpha$; see Figure 11(b).) Also, the sudden drop in stiffness occurs earlier with increasing values of $c$, consistent with the fact that $\alpha_{c r}$ is smaller for higher values of $c$ (see Figure 5(b)). On the other hand, Figures 13(c) and (d) show plots for the relevant Cauchy stress components and the particle rotation $\phi^{(\mathrm{I})}$, respectively, as functions of $\gamma$, for a fixed stretch $(\alpha=2)$. We observe from Figure 13(c) that the "soft" regime (i.e., the values of $\gamma$ for which the stress component $T_{12}$ is zero, i.e., $\left.\left[-\gamma_{c r}^{C}, \gamma_{c r}^{C}\right]\right)$ becomes larger with increasing $c$, consistent with the fact that $\gamma_{c r}^{C}$ is larger for higher values of $c$. Finally, it is noted from Figure 13(d) that the rotation of the fibers depends on $c$ in the relaxed domain $\left[-\gamma_{c r}^{C}, \gamma_{c r}^{C}\right]$, but interestingly it is otherwise independent of $c$, as predicted by the principal solution (see equation (41)).

Figure 14 presents plots for the relaxed estimates for the response of the fiberreinforced neo-Hookean elastomers for several fiber aspect ratios $(w=1,2,5,10)$, and a fixed volume fraction ( $c=0.3$ ). Figures $14(\mathrm{a})$ and (b) show plots for the normal stress difference $S$ and the particle rotation $\phi^{(\mathrm{I})}$, respectively, as functions of $\alpha$ for aligned loadings $(\gamma=0)$. We see from these figures that the effect of increasing $w$ on the macroscopic response of the composite is similar to the effect of increasing $c$ in that the composite becomes stiffer initially, and undergoes sharper drops in stiffness with increasing values of $w$. However, the post-bifurcation stresses at sufficiently large values of the deformation $\alpha$ also become smaller with increasing values of $w$. In addition, it should be noted that the special case $w=1$ corresponds to circular fibers, which do not rotate under this type of loading, and the relaxed and principal solutions are identical in this case. Next, Figures 14(c) and (d) show plots for the normal difference and shear Cauchy stresses, and the particle rotation $\phi^{(\mathrm{I})}$, respectively, as functions of $\gamma$, for a fixed stretch $(\alpha=2)$. It can be seen that the stresses are flat in the relaxed region $\left[-\gamma_{c r}^{C}, \gamma_{c r}^{C}\right]$, so that the corresponding mod- 


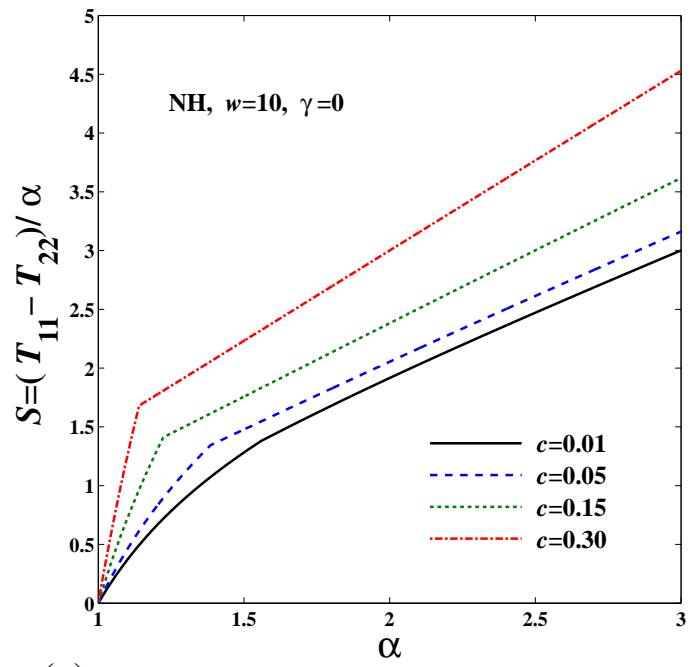

(a)

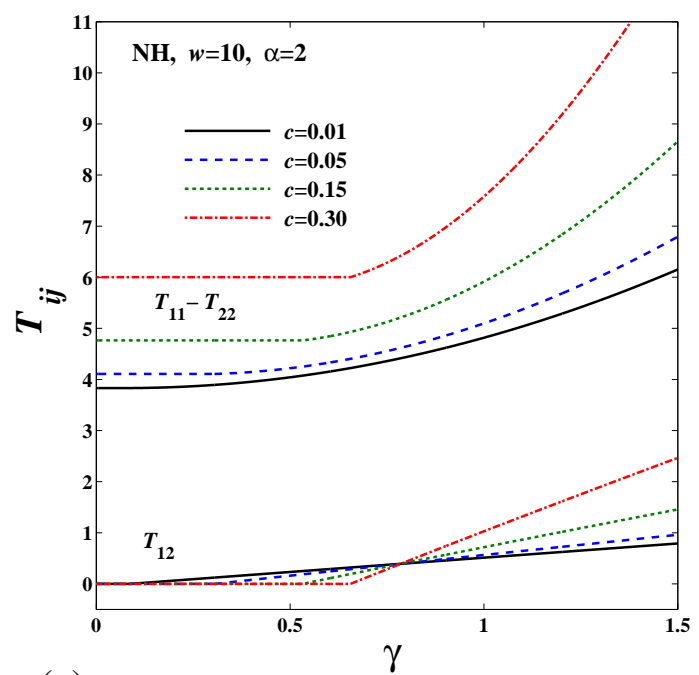

(c)

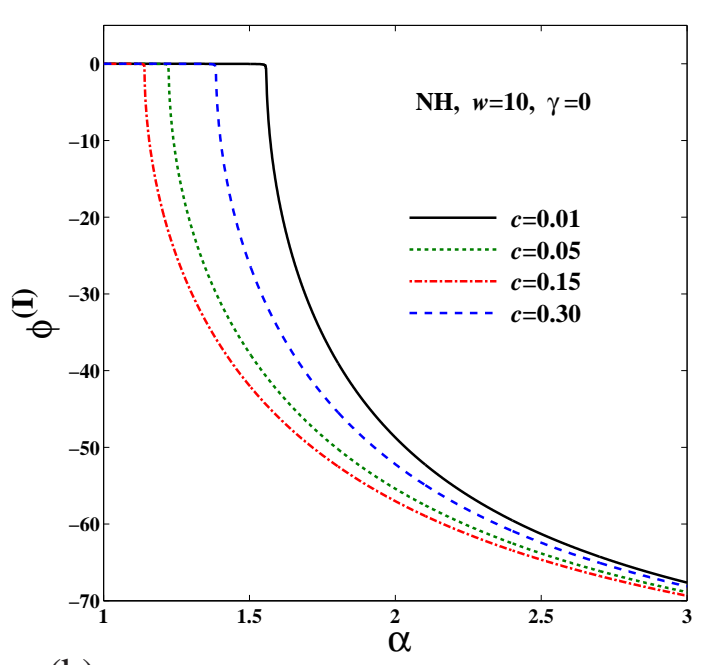

(b)

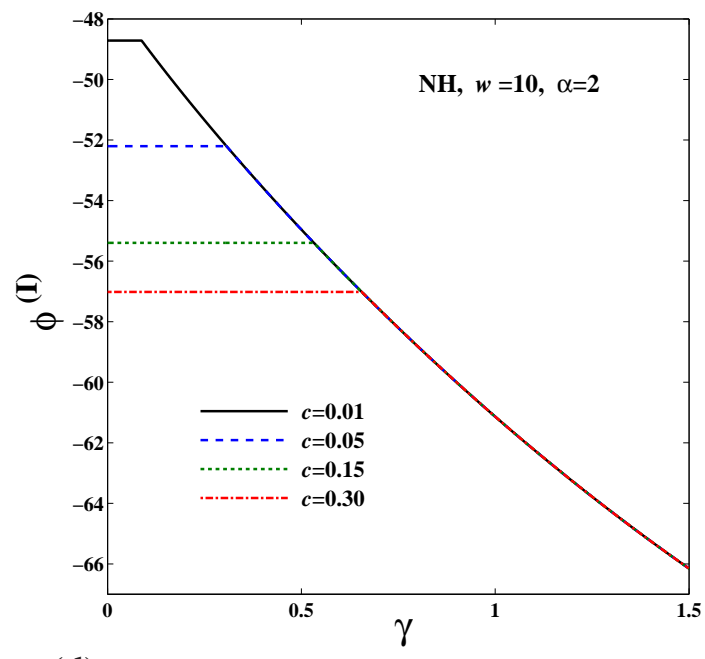

(d)

Figure 13: Relaxed estimates for the behavior of fiber-reinforced neo-Hookean elastomers with $w=10$ and various volume fractions $c$. Parts (a) and (b) show results for the normal stress difference $S=\left(T_{11}-T_{22}\right) / \alpha$ and the total rotation of the fibers in layer I, $\phi^{\mathrm{I}}$, respectively, as functions of $\alpha$, for aligned loadings $(\gamma=0)$. Parts (c) and (d) show results for the stress components $T_{i j}$, and the fiber rotation $\phi^{\mathrm{I}}$, respectively, as functions of $\gamma$, for a fixed value $\alpha=1.4$. 


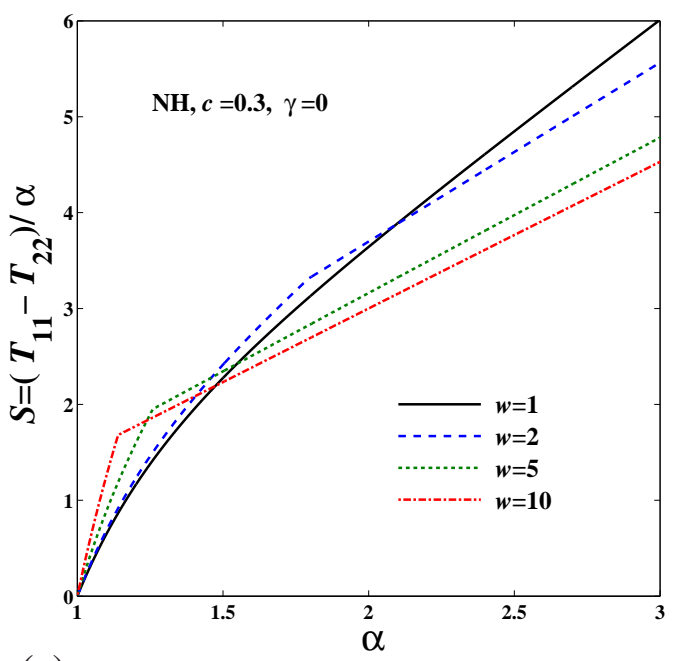

(a)

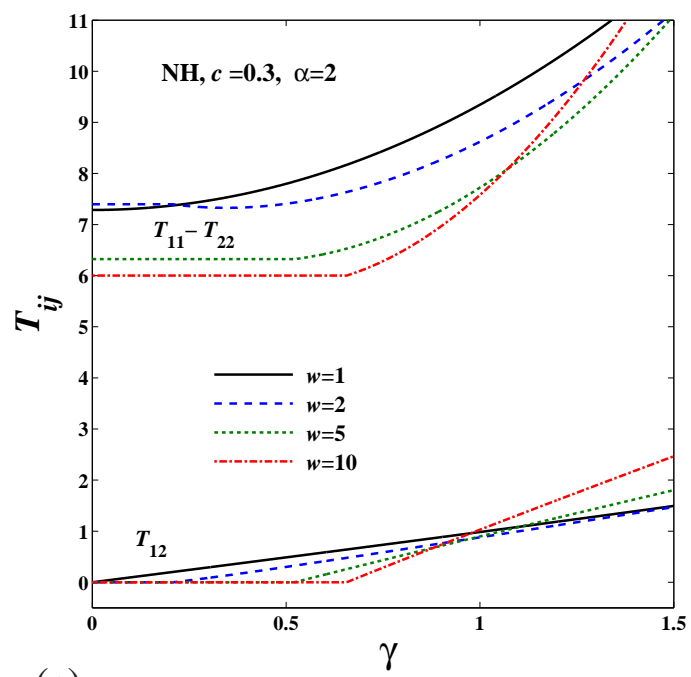

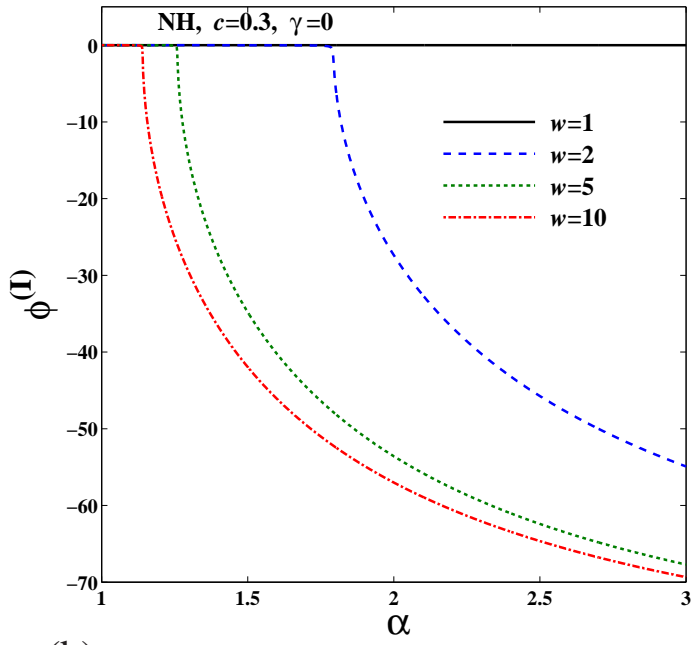

(b)

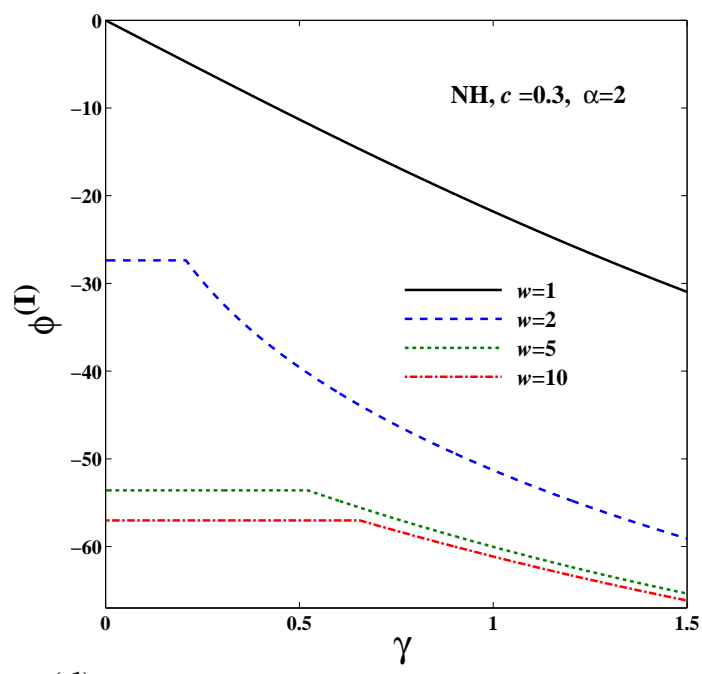

(d)

Figure 14: Relaxed estimates for the macroscopic and microscopic response of fiber-reinforced, neo-Hookean elastomers with $c=0.3$ and various aspect ratios $w$. Parts (a) and (b) show results for the normal stress difference $S=\left(T_{11}-T_{22}\right) / \alpha$ and the total rotation of the fibers in layer I, $\phi^{\mathrm{I}}$, respectively, for aligned loadings ( $\gamma=0$ ), as functions of $\alpha$. Parts (c) and (d) show results for the stress components $T_{i j}$, and the rotation of the fibers $\phi^{\mathrm{I}}$, respectively, as functions of $\gamma$, for a fixed stretch $\alpha=1.4$. 

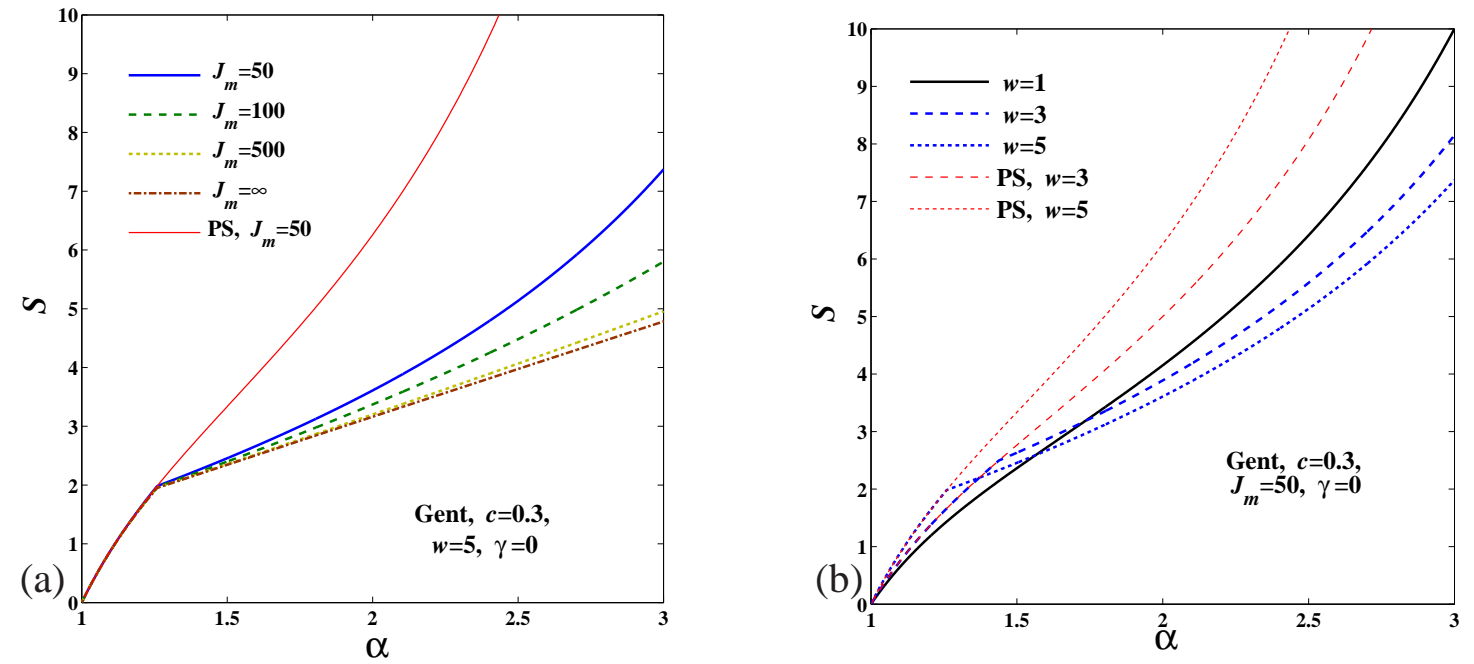

Figure 15: Relaxed estimates for the stress measure $S=\left(T_{11}-T_{22}\right) / \alpha$, as functions of $\alpha$, in fiber-reinforced, Gent elastomers with $c=0.3$ subjected to aligned loadings $(\gamma=0)$. Part (a) shows results for $w=5$ and various values of the lock-up parameter $J_{m}$. Part (b) shows results for $J_{m}=50$ and several aspect ratios $w$. The corresponding principal estimates are included for some plots for comparison.

uli vanish identically. This is consistent with the fact that the shear strain $\gamma$ in the relaxed region is entirely accommodated by the movement of the internal boundaries between the layers with different fiber orientations, leading to perfectly soft (liquid-like) response for transverse shear (in this region). On the other hand, the stresses are seen to increase with increasing values of $\gamma$ beyond the relaxed region $\left(\gamma>\gamma_{c r}^{C}\right)$. In addition, it is remarked that the size of the relaxed region increases with increasing values of $w$, while it vanishes for $w=1$.

Finally, Figure 15 presents plots for the relaxed and principal solutions for the stress measure $S$ in fiber-reinforced Gent-type elastomers with fixed volume fraction $c=0.3$. Figure 15(a) shows results for several values of the matrix strainlocking parameter $\left(J_{m}=50,100,500\right.$, and $\left.\infty\right)$, at the fixed aspect ratio $w=5$. It is recalled that the case $J_{m} \rightarrow \infty$ corresponds to an incompressible neo-Hookean matrix. Also, the corresponding principal solution for the case of $J_{m}=50$ is included for comparison purposes. The plots in Figure 15(a) show that the critical stretch $\alpha_{c r}$ is essentially independent of the matrix strain-locking parameter $J_{m}$. We further observe, by comparing the relaxed and principal solutions for the case of $J_{m}=50$, that the relaxed solution locks up at much larger strains than the principal solution. On the other hand, Figure 15(b) presents results for fiber-reinforced 
Gent elastomers with fiber aspect ratios $w=1,3$, and 5 and fixed strain-locking parameter $J_{m}=50$. As can be seen from this figure, the relaxed solution suggests that the locking strain for the Gent composites increases with the fiber aspect ratio, in contrast with the corresponding predictions from the principal solution that the composites with higher aspect ratio lock up at smaller strains. Also, consistent with observations made for the neo-Hookean composites in the context of Figure 14(a), larger aspect ratios have lower reinforcing effects on the macroscopic response of the composite beyond the critical stretch $\alpha_{c r}$.

\section{Concluding remarks}

In this work, we have constructed the "relaxed" stored-energy function for a class of anisotropic composite materials consisting of random distributions of aligned rigid fibers of elliptical cross section in a soft elastomeric matrix, which are subjected to general plane strain loading conditions. The starting point was the "mesoscopic" estimate of Lopez-Pamies and Ponte Castañeda (2006b) for the energy for this class of hyperelastic composite materials, which is known to lose strong ellipticity by the development of shear band instabilities under certain types of loadings. This energy function is therefore not quasiconvex, suggesting that domain microstructures may develop leading to the relaxation of the energy. The relaxation of the energy was accomplished by first computing the rank-one convex envelope of the "mesoscopic" estimate of Lopez-Pamies and Ponte Castañeda (2006b), and then showing that it is polyconvex and therefore quasiconvex. When sufficiently large compressive strains are applied along the long axes of the fibers, the composite undergoes a shear-band instability, giving way to a "striped domain" microstructure consisting of layers with alternating fiber orientations-the fibers rotating collectively and abruptly away from the direction of compression to relieve the high compressive stresses. The formation of the striped domains can be viewed as a symmetry-breaking transformation, where the symmetry of the microstructures goes from a "nematic" state, associated with the initially perfect alignment of the fibers, to a "smectic" state corresponding to the development of layers with alternating (fixed within the layers) fiber orientations. In turn, the relaxation of the energy can be thought of as a stress-driven "phase transition," and associated with it, there are also "soft modes" of deformation. Thus, in the relaxed or post-bifurcation regime, the composite can be deformed in shear transverse to the fibers without increasing the value of the stress, simply by varying the relative volume fractions of the layers with different fiber orientations. In other words, the elastic modulus in transverse shear is zero (i.e., the material response is liquid-like 
in this mode). On the other hand, the incremental elastic modulus of the composite under compression in the direction the fibers undergoes a finite drop in magnitude at the onset of the instability, but it remains a finite positive number. Away from this regime of deformations, the relaxed energy agrees exactly with the mesoscopic estimate of Lopez-Pamies and Ponte Castañeda (2006b) for the energy. In other words, the composite does not find it advantageous from an energetic point of view to form microstructures (i.e., to break itself up into striped domains to lower its overall energy), and the deformation in the composite is macroscopically uniform, consistent with the applied boundary conditions. In particular, for small deformations, the relaxed response agrees with the corresponding linearelastic theory of Ponte Castañeda and Willis (1995), from which the mesoscopic estimate of Lopez-Pamies and Ponte Castañeda (2006b) was derived by means of the second-order linear comparison homogenization method (Lopez-Pamies and Ponte Castañeda, 2006a). In this work, the effect of the fiber volume fraction and aspect ratio on the macroscopic response and associated instabilities were also explored, and found to be important.

It is interesting to comment here briefly on the similarities and differences of the short-fiber-reinforced elastomers (SFREs) investigated in this work and the liquid crystal elastomers (LCEs) considered elsewhere (Blandon et al., 1993; Warner and Terentjev, 1996; DeSimone and Dolzmann, 2000, 2002). Both of these systems have nematic order and appear to undergo similar instabilities, with the instabilities giving way to striped domains. However, at a fundamental level, SFREs and LCEs are genuinely different material systems. The "fibers" in the LCEs are mesogens, which are small compared to the cross-linking distances in the elastomer "matrix," and for this reason such fibers are free to rotate in the matrix without direct interference from the surrounding elastic chain network, giving these materials nominally isotropic response. On the other hand, the fibers in the SFREs are assumed to be large compared to the cross-linking distances in the elastomer matrix, so that the fibers interact strongly with the surrounding elastomer matrix, leading to anisotropic response. For these reasons, the mesoscopic energies for the two systems have been obtained by different means. The energies of the LCEs have been obtained using the molecular theory of rubber elasticity (Blandon et al., 1993), whereas those of the SFREs have made use of homogenization techniques for continuum hyperelastic models (Lopez-Pamies and Ponte Castañeda, 2006a,b). (It should be emphasized that the volume fraction and aspect ratio of the "fibers" do not appear explicitly in the energy expressions for the LCEs, the pertinent microstructural variable being the backbone anisotropy parameter in this case.) Not surprisingly, the resulting relaxed energies are also 
different. Most strikingly, the relaxed region of the LCEs is perfectly soft (all shear moduli vanish in the relaxed regime) and includes the reference configuration, while for the SFREs the stress-free reference configuration is not included in the relaxed regime, and the response of the material in this regime is not perfectly soft. As mentioned above, the material exhibits anisotropic response in this regime, with one shear modulus that vanishes and another that does not. At a more fundamental level, there is a consistent thermodynamic theory for the LCEs, with a high-temperature isotropic state, consisting of randomly oriented mesogens, and spontaneous deformations are possible by changes in temperature (Golubović and Lubensky, 1989; Warner and Terentjev, 1996). On the other hand, the SFREs are expected to be insensitive to changes in temperature (as long as the matrix remains in the rubbery state) and in this sense the stress-driven creation of striped domains is not a true phase transformation (in the thermodynamic sense). Nevertheless, the partial similarities between the two types of material systems are striking and suggest further investigation of the SFREs and their possible links to the LCEs.

Among issues for further study, it is important to recall that this work was concerned exclusively with a two-dimensional model problem, and it would be of practical and theoretical interest to also consider elastomers reinforced by threedimensional fibers under general loadings conditions. In this context, it is relevant to mention that mesoscopic estimates for the response for random distributions of aligned spheroidal fibers in a soft elastomer matrix have been given recently by Avazmohammadi and Ponte Castañeda (2014a,b), who also show that such estimates can develop shear band instabilities, albeit of a more complex nature. In addition, these estimates and their associated instabilities are different for prolate and oblate fibers. It would therefore be desirable to investigate the relaxation of these energies for the prolate and oblate cases. In particular, it will be interesting to see whether such a relaxation still corresponds to the rank-one convexification of the energy, and if so how many layering operations are required. In addition, it should also be recalled here that loss of ellipticity has also been found for corresponding mesoscopic estimates of Lopez-Pamies and Ponte Castañeda (2007a,b) for the stored-energy functions of porous elastomers with random distributions of initially spherical holes. Such material systems are isotropic and compressible and may provide additional test cases to further explore the use of the theory of relaxation in the context of more general hyperelastic composite materials. Naturally, experimental work will be crucial in determining the physical relevance of these theoretical predictions. 


\section{Acknowledgements}

This material is based upon work supported by the National Science Foundation, which began under Grant No. CMMI-1068769 and was completed under Grant No. DMS-1108847. The writing of the paper was completed while PPC was visiting at the University of Stuttgart with the support of the Humboldt Research Award.

\section{Appendix A. Partial derivatives of $\widehat{\Phi}$ with respect to $\lambda$ and $\theta$}

The first derivatives of the effective strain-energy function, defined in (38), with respect to kinematical variables $\lambda$ are $\theta$ are given by

$$
\begin{aligned}
\frac{\partial \widehat{\Phi}}{\partial \lambda} & =\frac{g^{\prime}}{(1-c) w \lambda^{2}}\left\{2 w \lambda^{3}+2 c\left(w^{2}+1\right) \lambda^{-1}\left(\lambda^{2}+1\right)\left(\lambda^{2}+2 \lambda \cos (\varphi)-1\right)\right. \\
& -2 c\left(w^{2}+1\right)\left(3 \lambda^{2}+1\right) \cos (\varphi)-2 c\left(w^{2}-1\right) \lambda^{-1}\left(\lambda^{4}+1\right) \sin (\varphi) \sin (\varphi-2 \theta) \\
& \left.+c d_{w}\left[\left(w^{2}+1\right) \sin (\varphi)-\left(w^{2}-1\right) \lambda \sin (2 \varphi-2 \theta)\right]-2 w \lambda^{-1}\right\}
\end{aligned}
$$

where $g^{\prime}=\mathrm{d} g(\hat{I}) / \mathrm{d} \hat{I}$, and

$$
d_{w}=\frac{\left(w^{2}-1\right)\left(\lambda^{4}-1\right) \sin (2 \varphi-2 \theta)-2\left(w^{2}+1\right) \lambda\left(\lambda^{2}+1\right) \sin (\varphi)}{\left(w^{2}+1\right) \lambda \cos (\varphi)-\left(w^{2}-1\right)\left(\lambda^{2}-1\right) \cos (2 \varphi-2 \theta)}
$$

The higher derivatives of $\widehat{\Phi}$ with respect to $\lambda$ and $\theta$ can also be explicitly obtained from the above expressions, together with the kinematical equation (41), but the results are not included here for brevity.

\section{Appendix B. Strong Ellipticity conditions for the reinforced elastomer}

In this appendix, we recall some results (Lopez-Pamies and Ponte Castañeda, 2006b) concerning the strong ellipticity conditions associated with the principal solution for the effective stored-energy function $\widehat{W}(\mathbf{F})$. In particular, we are interested in developing the specific conditions under which the effective storedenergy function (38) of the fiber-reinforced elastomers can lose strong ellipticity. For simplicity of notation, we drop here the hat . over the elasticity tensor $\mathbf{L}$, as 
given by expression (49). We begin by recalling that the strong ellipticity condition (i.e., expression (11) with the inequality $\geq$ replaced by a strict inequality $>$ ) may be rewritten in terms of the instantaneous (i.e., the push forward to the current configuration) elasticity tensor $\mathbf{L}^{c}$ (Ogden, 1997) via

$$
m_{i} n_{j} L_{i j k l}^{c} m_{k} n_{l}>0, \quad \text { for all vectors } \mathbf{m} \text { and } \mathbf{n},
$$

where $\mathbf{L}^{c}$ and $\mathbf{n}$ are defined by

$$
L_{i j k l}^{c}=J^{-1} L_{i p k q} F_{j p} F_{l q}, \quad \mathbf{n}=J \mathbf{F}^{-T} \mathbf{N} .
$$

For the case of incompressible materials, condition (B.1) is still valid provided that the two arbitrary vectors $\mathbf{n}$ and $\mathbf{m}$ satisfy the requirement that $\mathbf{n} \cdot \mathbf{m}=0$ (which is a consequence of the incompressibility constraint.) Accordingly, the strong ellipticity condition (B.1), when specialized to the plane-strain loading (31), can be expressed as

$$
\begin{aligned}
L_{2121}^{c}\left(\frac{n_{1}}{n_{2}}\right)^{4} & -2\left(L_{1121}^{c}-L_{2221}^{c}\right)\left(\frac{n_{1}}{n_{2}}\right)^{3}+2\left(L_{1112}^{c}-L_{2212}^{c}\right)\left(\frac{n_{1}}{n_{2}}\right)+L_{1212}^{c} \\
& +\left[L_{1111}^{c}+L_{2222}^{c}-2\left(L_{1122}^{c}+L_{1221}^{c}\right)\right]\left(\frac{n_{1}}{n_{2}}\right)^{2}>0,
\end{aligned}
$$

which is a fourth-order polynomial in the ratio $n_{1} / n_{2}$. The coefficients of this polynomial are bounded traces of the the instantaneous elasticity tensor $\mathbf{L}^{c}$. They can be rewritten in terms of $\widehat{\Phi}$ and its first and second derivatives with respect to $\lambda$ and $\theta$ by making use of the objectivity of $\widehat{W}$, together with expression (B.2), and of the Lagrangian coordinates (see Fig. 2(b)). It follows that loss of ellipticity for the homogenized rigidly reinforced, incompressible elastomer (40) under planestrain loading (31) occurs when the polynomial equation

$$
\begin{aligned}
& \lambda^{3}\left[\lambda\left(\lambda^{4}-1\right) \frac{\partial \widehat{\Phi}}{\partial \lambda}+\frac{\partial^{2} \widehat{\Phi}}{\partial \theta^{2}}\right] \eta^{4}+2 \lambda\left[\left(3 \lambda^{4}+1\right) \frac{\partial \widehat{\Phi}}{\partial \theta}-\lambda\left(\lambda^{4}-1\right) \frac{\partial^{2} \widehat{\Phi}}{\partial \lambda \partial \theta}\right] \eta^{3} \\
& +\left[\lambda\left(\lambda^{4}-1\right)^{2} \frac{\partial^{2} \widehat{\Phi}}{\partial \lambda^{2}}-2\left(\lambda^{4}-1\right) \frac{\partial \widehat{\Phi}}{\partial \lambda}-2 \lambda^{3} \frac{\partial^{2} \widehat{\Phi}}{\partial \theta^{2}}\right] \eta^{2} \\
& +2 \lambda\left[\lambda\left(\lambda^{4}-1\right) \frac{\partial^{2} \widehat{\Phi}}{\partial \lambda \partial \theta}-\left(\lambda^{4}+3\right) \frac{\partial \widehat{\Phi}}{\partial \theta}\right] \eta+\left(\lambda^{4}-1\right) \frac{\partial \widehat{\Phi}}{\partial \lambda}+\lambda^{3} \frac{\partial^{2} \widehat{\Phi}}{\partial \theta^{2}}=0
\end{aligned}
$$


first has a real root $\eta$. It is also remarked that $\eta=\cot (\Gamma-\theta-\psi)$, where $\Gamma$ is the angle that the normal vector $\mathbf{n}$ (in the current configuration) makes with the $\mathbf{e}_{1}$ axis precisely at the moment of the band initiation. Then, making use of the energy form (40) and relations (34)-(36), the above equation can be used to find the critical deformation in the $(\alpha, \gamma)$-space at which $\widehat{W}$ loses strong ellipticity for the first time.

For the special case of (plane strain) macroscopic loadings that are aligned with the fiber axes $\left\{\mathbf{e}_{i}\right\}$ and thus characterized by the condition $\gamma=0$ in (31) (or, equivalently, the condition $\theta=\psi=0$ in (32)), so that $\alpha=\lambda$, it can be shown that the strong ellipticity condition (B.3) reduces to the following inequalities

$$
L_{1212}^{c}>0, \quad L_{2121}^{c}>0, \quad L_{1111}^{c}+L_{2222}^{c}-2\left(L_{1122}^{c}+L_{1221}^{c}\right)+2 \sqrt{L_{1212}^{c} L_{2121}^{c}}>0 .
$$

Employing the expressions for these specific moduli traces in terms of $\widehat{\Phi}$, inequalities (B.5) can be respectively rewritten as

$$
\begin{aligned}
& \left(\lambda^{4}-1\right) \frac{\partial \widehat{\Phi}}{\partial \lambda}+\lambda^{3} \frac{\partial^{2} \widehat{\Phi}}{\partial \theta^{2}}>0 \\
& \lambda\left(\lambda^{4}-1\right) \frac{\partial \widehat{\Phi}}{\partial \lambda}+\frac{\partial^{2} \widehat{\Phi}}{\partial \theta^{2}}>0 \\
& \frac{1}{2}\left[\lambda\left(\lambda^{4}-1\right)^{2} \frac{\partial^{2} \widehat{\Phi}}{\partial \lambda^{2}}-2\left(\lambda^{4}-1\right) \frac{\partial \widehat{\Phi}}{\partial \lambda}-2 \lambda^{3} \frac{\partial^{2} \widehat{\Phi}}{\partial \theta^{2}}\right] \\
& +\lambda \sqrt{\lambda}\left[\lambda\left(\lambda^{4}-1\right)^{2}\left(\frac{\partial \widehat{\Phi}}{\partial \lambda}\right)^{2}+\lambda^{3}\left(\frac{\partial^{2} \widehat{\Phi}}{\partial \theta^{2}}\right)^{2}+\left(\lambda^{8}-1\right) \frac{\partial \widehat{\Phi}}{\partial \lambda} \frac{\partial^{2}}{\partial \theta^{2}}\right]^{\frac{1}{2}}>0
\end{aligned}
$$

where all derivatives are evaluated at $\theta=0$. By explicit computations, it can be demonstrated that (for the energy function (40)) the first inequality (corresponding to $L_{1212}^{c}>0$ ) is always violated before the second inequality (corresponding to $\left.L_{2121}^{c}>0\right)$ at a critical stretch, denoted by $\alpha_{c r}$. In addition, it is observed that the last inequality is not violated up to very large stretches.

\section{Appendix C. Polyconvexity of $R \widehat{W}(\mathbf{F})$}

For our purposes here, it is useful to introduce the invariants

$$
i_{1}=\sqrt{C_{11}}=\left|\mathbf{F} \mathbf{N}_{f}\right|=\sqrt{I_{2}}, \quad i_{2}=\sqrt{C_{22}}=\left|\mathbf{F}^{a} \mathbf{N}_{f}\right|=\sqrt{I_{1}-I_{2}},
$$


where it is recalled that $\mathbf{C}=\mathbf{F}^{T} \mathbf{F}$ is the right Cauchy-Green tensor and $\mathbf{F}^{a}$ is the adjugate of $\mathbf{F}$. For general plane strain deformations, $i_{1}, i_{2}$ and $J$ are equivalent to the set of invariants $I_{1}, I_{2}$ and $J$, defined expression (23). In terms of $i_{1}, i_{2}$ and $J$, the invariants $\alpha$ and $\gamma$, defined by expressions (28) and (29), can be alternatively written

$$
\begin{aligned}
& \alpha=\frac{i_{1}}{\sqrt{J}}, \\
& \gamma=\left\{\begin{array}{l}
\sqrt{\frac{i_{2}^{2}}{J}-\frac{J}{i_{1}^{2}}}, \quad \text { if } \quad F_{11} F_{12}+F_{21} F_{22} \geq 0, \\
-\sqrt{\frac{i_{2}^{2}}{J}-\frac{J}{i_{1}^{2}}}, \quad \text { if } \quad F_{11} F_{12}+F_{21} F_{22} \leq 0 .
\end{array}\right.
\end{aligned}
$$

In connection with this last expression, it is important to remark that the condition

$$
\frac{i_{1} i_{2}}{J} \geq 1
$$

must be enforced. This condition follows easily by noting that

$$
\left(\frac{i_{1} i_{2}}{J}\right)^{2}=\frac{\left(F_{11}^{2}+F_{21}^{2}\right)\left(F_{22}^{2}+F_{12}^{2}\right)}{\left(F_{11} F_{22}-F_{12} F_{21}\right)^{2}}=1+\left(\frac{F_{11} F_{12}+F_{22} F_{21}}{F_{11} F_{22}-F_{12} F_{21}}\right)^{2} \geq 1,
$$

and recalling that $i_{1}, i_{2}$ and $J$ are all non-negative. (Of course, an analogous condition must be satisfied by $I_{1}, I_{2}$ and $J$ in the earlier expressions for $\gamma$.)

Next, we define the function $\breve{W}$ such that

$$
\check{W}(\mathbf{F})=\widehat{\Psi}^{* *}\left(\alpha=\frac{i_{1}}{\sqrt{J}}, \gamma= \pm \sqrt{\frac{i_{2}^{2}}{J}-\frac{J}{i_{1}^{2}}}\right) .
$$

It is emphasized that $\breve{W}$ agrees exactly with $\widehat{\Psi}^{* *}$, as defined earlier in the context of expression (65) for $R \widehat{W}$, when $J=\operatorname{det} \mathbf{F}=1$, but is well-defined for more general values of $\mathbf{F}$ with $J=\operatorname{det} \mathbf{F}>0$. Therefore, we can also write $R \widehat{W}$ in the alternative form

$$
R \widehat{W}(\mathbf{F})=\check{W}(\mathbf{F})+K(\operatorname{det} \mathbf{F}) .
$$

Noting that $K$ is convex in $J=\operatorname{det} \mathbf{F}$, and therefore polyconvex, to show that $R \widehat{W}$ is polyconvex, it suffices to demonstrate that $\breve{W}$ is convex in $\mathbf{F}$ for values of $\mathbf{F}$ such that $J=\operatorname{det} \mathbf{F}=1$. 
For this purpose, we introduce the function $g$ such that

$$
g\left(i_{1}, i_{2}\right)=\widehat{\Psi}^{* *}\left(\alpha=i_{1}, \gamma= \pm \sqrt{i_{2}^{2}-1 / i_{1}^{2}}\right)
$$

and we show (see, for example Steigmann, 2003) that $\check{W}$ is convex (for values of $\mathbf{F}$ with $J=\operatorname{det} \mathbf{F}=1$ ) if the above-defined function $g$ satisfies the conditions:

(a) $g$ is a convex function of $i_{1}, i_{2}$ jointly, and

(b) $g$ a non-decreasing function of $i_{1}$ and $i_{2}$.

With this goal in mind, we let $\Delta i_{1}=i_{1}(\mathbf{F}+\Delta \mathbf{F})-i_{1}(\mathbf{F})$ and similarly for $\Delta i_{2}$, and we consider the Taylor expansion of $g\left(i_{1}+\Delta i_{1}, i_{2}+\Delta i_{2}\right)$ about $\left(i_{1}, i_{2}\right)$

$g\left(i_{1}+\Delta i_{1}, i_{2}+\Delta i_{2}\right)=g\left(i_{1}, i_{2}\right)+g_{, i_{1}}\left(\Delta i_{1}\right)+g_{, i_{2}}\left(\Delta i_{2}\right)+\frac{1}{2} \mathbf{V} \cdot\left(D^{2} g\right) \mathbf{V}^{T}+o\left(|\mathbf{V}|^{3}\right)$,

where $\mathbf{V}=\left(\Delta i_{1}, \Delta i_{2}\right)$ is a $1 \times 2$ vector and $D^{2} g$ is the $2 \times 2$ Hessian matrix (the matrix of second-order derivatives) of the function $g\left(i_{1}, i_{2}\right)$. In addition, the comma subscripts denote partial derivatives with respect to subsequent subscripts, and such derivatives are evaluated at $\left(i_{1}, i_{2}\right)$. Then, property $(a)$ in (C.9) implies that the second-order term in the above expansion is non-negative, and it follows that

$$
\begin{aligned}
\check{W}(\mathbf{F}+\Delta \mathbf{F})-\check{W}(\mathbf{F}) & =g\left(i_{1}+\Delta i_{1}, i_{2}+\Delta i_{2}\right)-g\left(i_{1}, i_{2}\right) \\
& \geq g_{, i_{1}}\left(\Delta i_{1}\right)+g_{, i_{2}}\left(\Delta i_{2}\right) .
\end{aligned}
$$

On the other hand, it is easy to show that $i_{1}$ and $i_{2}$ are convex functions of $\mathbf{F}$, so that making use of Taylor expansion of $i_{1}+\Delta i_{1}$ and $i_{2}+\Delta i_{2}$ about $i_{1}$ and $i_{2}$, respectively, it similarly follows that

$$
\Delta i_{1} \geq\left(i_{1}\right)_{, \mathbf{F}} \cdot \Delta \mathbf{F}, \quad \Delta i_{2} \geq\left(i_{2}\right)_{, \mathbf{F}} \cdot \Delta \mathbf{F} .
$$

Combining the above two inequalities together with property (b) in (C.9) (implying that $g_{, i_{1}}$ and $g_{, i_{2}}$ are non-negative), we conclude that

$$
\check{W}(\mathbf{F}+\Delta \mathbf{F})-\check{W}(\mathbf{F}) \geq\left[g_{, i_{1}}\left(i_{1}\right)_{, \mathbf{F}}+g_{, i_{2}}\left(i_{2}\right)_{, \mathbf{F}}\right] \cdot \Delta \mathbf{F},
$$

which shows that $\check{W}$ is convex in $\mathbf{F}$.

It now remains to show that the function $g$, as defined by (C.8), satisfies the conditions (C.9). In this regard, it is recalled that the domain of interest is given 


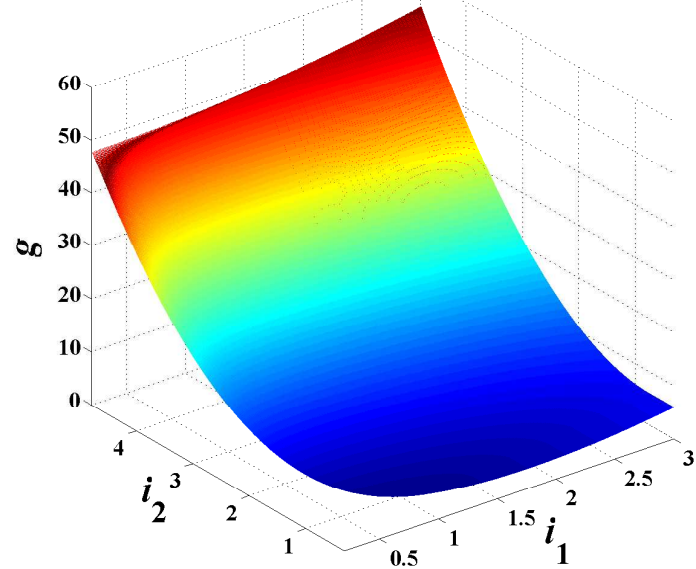

(a)

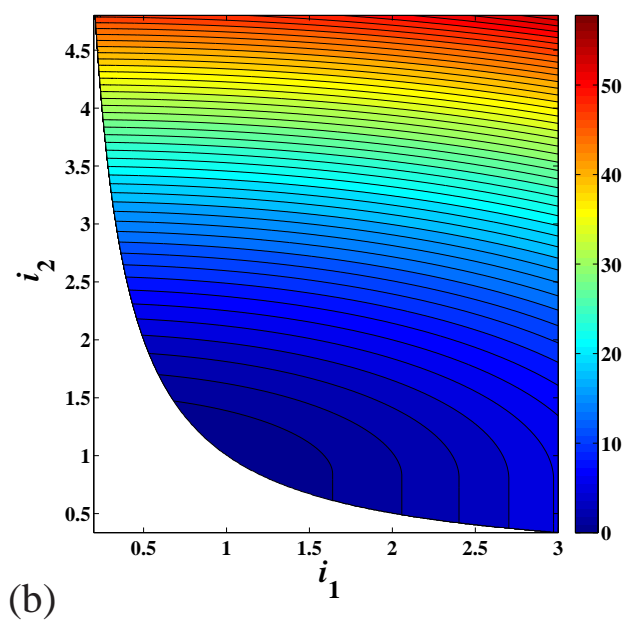

(b)

Figure C.16: (a) 3-D landscape plot of $g$. (b) Corresponding contour plot.

by $i_{2} \geq 1 / i_{1}$ and $i_{1}>0$, which is a convex domain. It is then straightforward to check (numerically) that the Hessian matrix, which is given by

$$
D^{2} g=\left(\begin{array}{ll}
g_{, i_{1} i_{1}} & g_{, i_{1} i_{2}} \\
g_{, i_{2} i_{1}} & g_{, i_{2} i_{2}}
\end{array}\right)
$$

is positive semi-definite, from which it follows that $g$ is convex in $i_{1}$ and $i_{2}$. It is also straightforward to check numerically that $g$ is non-decreasing in $i_{1}$ and $i_{2}$. For instance, Fig. C.16(a) and (b) respectively show a 3-D landscape and the corresponding contour plot of $g\left(i_{1}, i_{2}\right)$, as a function of $i_{1}$ and $i_{2}$, for reinforced elastomers with $w=10$ and $c=0.3$.

\section{References}

Abeyaratne, R., Triantafyllidis, N., 1984. An investigation of localization in a porous elastic material using homogenization theory. ASME J. Appl. Mech. 51, 481-486.

Agoras, M., Lopez-Pamies, O., Ponte Castañeda, P., 2009a. A general hyperelastic model for incompressible fiber-reinforced elastomers. J. Mech. Phys. Solids 57, 268-286. 
Agoras, M., Lopez-Pamies, O., Ponte Castañeda, P., 2009b. Onset of macroscopic instabilities in fiber-reinforced elastomers at finite strain. J. Mech. Phys. Solids $57,1828-1850$.

Avazmohammadi, R., Ponte Castañeda, P., 2013. Tangent second-order estimates for the large-strain, macroscopic response of particle-reinforced elastomers. J. Elasticity 112, 139-183.

Avazmohammadi, R., Ponte Castañeda, P., 2014. On the macroscopic response, microstructure evolution, and macroscopic stability of short-fiber-reinforced elastomers at finite strains: I-Analytical results. Phil. Mag. 94, 1031-1067.

Avazmohammadi, R., Ponte Castañeda, P., 2014. On the macroscopic response, microstructure evolution, and macroscopic stability of short-fiber-reinforced elastomers at finite strains: II-Representative examples. Phil. Mag. 94, 1068 1094.

Ball, J.M., 1977. Convexity conditions and existence theorems in nonlinear elasticity. Arch. Rational Mech. Anal. 63, 337-403.

Ball, J.M., James, R.D., 1986. Fine phase mixtures as minimizers of energy. Arch. Rational Mech. Anal. 94, 307-334.

Bertoldi, K., Boyce, M.C., Deschanel, S., Prange, S.M., Mullin, T., 2007. Mechanics of deformation-triggered pattern transformations and superelastic behavior in periodic elastomeric structures. J. Mech. Phys. Solids 56, 2642-2668.

Blandon, P., Terentjev, E.M., Warner, M., 1993. Transitions and instabilities in liquid-crystal elastomers. Phys. Rev. E 47, R3838-R3840.

Bouchart, V., Brieu, M., Bhatnagar, N. Kondo, D., 2010. A multiscale approach of nonlinear composites under finite deformation: Experimental characterization and numerical modeling. Int. J. Solids Struct. 47, 1737-1750.

Braides, A.,1985. Homogenization of some almost periodic coercive functionals. Rend. Accad. Naz. XL 9, 313-322.

Brun, M., Lopez-Pamies, O., Ponte Castañeda, P., 2007. Homogenization estimates for fiber-reinforced elastomers with periodic microstructures. Int. J. Solids Struct. 44, 5953-5979. 
Chen, H., Liu, Y., Zhao, X., Lanir, Y., Kassab, G. S., 2011. A micromechanics finite-strain constitutive model of fibrous tissue. J. Mech. Phys. Solids 59, 18231837.

Ciarlet, P., 1988. Mathematical elasticity, Vol. I: Three-dimensional elasticity. North-Holland, Amsterdam, The Netherlands.

Conti, S., 2008. Quasiconvex functions incorporating volumetric constraints are rank-one convex. J. Math. Pures Appl. 90, 15-30.

Dacorogna, B., 2007. Direct methods in the calculus of variations. (Applied Mathematical Sciences, Vol. 78). Springer, New York.

deBotton, G., 2005. Transversely isotropic sequentially laminated composites in finite elasticity. J. Mech. Phys. Solids 53, 1334-1361.

deBotton, G., Hariton, I., Socolsky, E.A., 2006. Neo-Hookean fiber-reinforced composites in finite elasticity. J. Mech. Phys. Solids 54, 533-559.

Desimone, A., 1993. Energy minimizers for large ferromagnetic bodies. Arch. Rational Mech. Anal. 125 (2), 99-143.

DeSimone, A., Dolzmann, G., 2000. Material instabilities in nematic elastomers. Physica D 136, 175-191.

DeSimone, A., Dolzmann, G., 2002. Macroscopic response of nematic elastomers via relaxation of a class of so (3)-invariant energies. Arch. Rational Mech. Anal. 161 (3), 181-204.

Gent, A., 1996. A new constitutive relation for rubber. Rubber Chem. Tech. 69, 59-61.

Geymonat, G., Müller, S., Triantafyllidis, N., 1993. Homogenization of nonlinearly elastic materials, microscopic bifurcation and macroscopic loss of rankone convexity. Arch. Rational Mech. Anal. 122, 231-290.

Golubović, L., Lubensky, T.C.., 1989. Nonlinear elasticity of amorphous solids. Phys. Rev. Lett. 63, 1082-1086.

Guo, Z.Y., Peng, X.Q., Moran, B., 2007. Large deformation response of a hyperelastic fibre reinforced composite: Theoretical model and numerical validation. Composites: Part A 38, 1842-1851. 
Hashin, Z., 1985. Large isotropic elastic deformation of composites and porous media. Int. J. Solids Struct. 21, 711-720.

Hill, R., 1972. On constitutive macro-variables for heterogeneous solids at finite strain. Proc. Roy. Soc. Lon. A 326, 131-147.

Honeker, C., Thomas, E., 1996. Impact of morphological orientation in determining mechanical properties in triblock copolymers. Chem. Mater. 8, 1702-1714.

Jimenez, F.L., Pellegrino, S., 2012. Folding of fiber composites with a hyperelastic matrix. Int. J. Solids Struct. 49, 395-407.

Kohn, R.V., Strang, G., 1986. Optimal design and relaxation of variational problems, II. Comm. Pure Appl. Math XXXIX, 139-182.

Lahellec, N., Mazerolle, F., Michel, J.C., 2004. Second-order estimate of the macroscopic behavior of periodic hyperelastic composites: theory and experimental validation. J. Mech. Phys. Solids 52, 27-49.

Lopez-Pamies, O., Garcia, R., Chabert, E., Cavaillé, J.-Y., Ponte Castañeda, P., 2008. Multiscale modeling of oriented thermoplastic elastomers with lamellar morphology. J. Mech. Phys. Solids 56, 3206-3223.

Lopez-Pamies, O., Ponte Castañeda, P., 2004. Second-order estimates for the macroscopic response and loss of ellipticity in porous rubbers at large deformations. J. Elasticity 76, 247-287.

Lopez-Pamies, O., Ponte Castañeda, P., 2006a. On the overall behavior, microstructure evolution, and macroscopic stability in reinforced rubbers at large deformations: I — Theory. J. Mech. Phys. Solids 54, 807-830.

Lopez-Pamies, O., Ponte Castañeda, P., 2006b. On the overall behavior, microstructure evolution, and macroscopic stability in reinforced rubbers at large deformations: II — Application. J. Mech. Phys. Solids 54, 831-863.

Lopez-Pamies, O., Ponte Castañeda, P., 2007a. Homogenization-based constitutive models for porous elastomers and implications for macroscopic instabilities: I-Analysis. J. Mech. Phys. Solids 55, 1677-1701.

Lopez-Pamies, O., Ponte Castañeda, P., 2007b. Homogenization-based constitutive models for porous elastomers and implications for macroscopic instabilities: II-Results. J. Mech. Phys. Solids 55, 1702-1728. 
Lopez-Pamies, O., Ponte Castañeda, P., 2009. Microstructure evolution in hyperelastic laminates and implications for overall behavior and macroscopic stability. Mech. Mater. 41, 364-374.

Marcellini, P., 1978. Periodic solutions and homogenization of nonlinear variational problems. Ann. Mat. Pura Appl. 4, 139-152.

Michel, J.C., Lopez-Pamies, O., Ponte Castañeda, P., Triantafyllidis, N., 2007. Microscopic and macroscopic instabilities in finitely strained porous elastomers. J. Mech. Phys. Solids 55, 900-938.

Michel, J.C., Lopez-Pamies, O., Ponte Castañeda, P., Triantafyllidis, N., 2010. Microscopic and macroscopic instabilities in finitely strained fiber-reinforced elastomers. J. Mech. Phys. Solids 58, 1776-1803.

Miehe, C., Schröder, J., Becker, M., 2002. Computational homogenization analysis in finite elasticity: material and structural instabilities on the micro- and macro-scales of periodic composites and their interaction. Comput. Methods Appl. Mech. Engrg. 191, 4971-5005

Moraleda, J., Segurado, J., Llorca, J., 2007. Finite deformation of porous elastomers: A computational micromechanics approach. Phil. Mag. 87, 5607-5627.

Moraleda, J., Segurado, J., Llorca, J., 2009. Finite deformation of incompressible fiber-reinforced elastomers: A computational micromechanics approach. J. Mech. Phys. Solids 57, 1596-1613.

Morrey, C., 1952. Quasiconvexity and the lower semicontinuity of multiple integrals. Pacific J. Math. 2, 25-53.

Müller, S., 1987. Homogenization of nonconvex integral functionals and cellular elastic materials. Arch. Rational Mech. Anal. 99, 189-212.

Mullin, T., Deschanel, S., Bertoldi, K., Boyce, M.C., 2007. Pattern transformation triggered by deformation. Phys. Rev. Lett. 99, 84301.

Nestorovic, M., Triantafyllidis, N., 2004. Onset of failure in finitely strained layered composites subjected to combined normal and shear loading. J. Mech. Phys. Solids, 52, 941-974.

O'Connor, J. E., 1977. Short-fiber-reinforced elastomer composites. Rubber Chem. Technol., 50, 945-958. 
Ogden, R., 1978. Extremum principles in non-linear elasticity and their application to composites-I. Theory. Int. J. Solids Struct. 14, 265-282.

Ogden, R., 1997. Non-linear Elastic Deformations. Dover, New York.

Ponte Castañeda, P., 1989. The overall constitutive behavior of nonlinearly elastic composites. Proc. R. Soc. Lond. A 422, 147-171.

Ponte Castañeda, P., 1991. The effective mechanical properties of nonlinear isotropic composites. J. Mech. Phys. Solids 39, 45-71.

Ponte Castañeda, P., 1996. Exact second-order estimates for the effective mechanical properties of nonlinear composite materials. J. Mech. Phys. Solids, $44,827-862$.

Ponte Castañeda, P., 2002. Second-order homogenization estimates for nonlinear composites incorporating field fluctuations. I - Theory. J. Mech. Phys. Solids $50,737-757$.

Ponte Castañeda, P., Suquet, P., 1998. Nonlinear composites. Adv. Appl. Mech. $34,171-302$.

Ponte Castañeda, P., Tiberio, E., 2000. A second-order homogenization method in finite elasticity and applications to black-filled elastomers. J. Mech. Phys. Solids 48, 1389-1411.

Ponte Castañeda, P., Willis, J.R., 1995. The effect of spatial distribution on the effective behavior of composite materials and cracked media. J. Mech. Phys. Solids 43, 1919-1951.

Racherla, V., Lopez-Pamies, O., Ponte Castañeda, P., 2010. Macroscopic response and stability in lamellar nanostructured elastomers with "oriented and "unoriented polydomain microstructures. Mech. Mater. 42, 451-468.

Spencer, A., 1984. Constitutive theory for strongly anisotropic solids. In Continuum Theory of the Mechanics of Fibre-Reinforced Composites (C.I.S.M. Vol. 282), Springer-Verlag, New York, pp. 1-32.

Steigmann, D., 2003. Frame-invariant polyconvex strain-energy functions for some anisotropic solids. Math. Mech. Solids 8, 497-506. 
Suquet, P., Ponte Castañeda, P., 1993. Small-contrast perturbation expansions for the effective properties of nonlinear composites. C.R. Acad. Sc. Paris II 317, $1515-1522$.

Triantafyllidis, N., Maker, B.N., 1985. On the comparison between microscopic and macroscopic instability mechanisms in a class of fiber-reinforced composites. J. Appl. Mech. 52, 794-800.

Triantafyllidis, N., Nestorovic, M., Schaard, M., 2006. Failure surfaces for finitely strained two-phase periodic solids under general in-plane loading. J. Appl. Mech. 73, 505-516.

Truesdell, C., Noll, W., 1965. The Non-Linear Field Theories of Mechanics. In Handbuch der Physik, Vol. III/3 (Ed. S. Flügge), Springer.

Warner, M., Terentjev, E.M., 1996. Nematic elastomers - a new state of matter?. Prog. Polym. Sci. 21, 853-891.

Willis, J.R., 1981. Variational and related methods for the overall properties of composites. Adv. Appl. Mech. 21, 1-78. 